\begin{tabular}{|c|l|}
\hline Title & Estimating the value of travel time and of travel time reliability in road networks \\
\hline Author(s) & Uchida, Kenetsu \\
\hline Citation & $\begin{array}{l}\text { Transportation Research Part B Methodological, 66, 129-147 } \\
\text { https://doi.org/10.1016/.trb.2014.01.002 }\end{array}$ \\
\hline Issue Date & 201408 \\
\hline Doc URL & http://hdl.handle.net/2115/57050 \\
\hline Type & article (author version) \\
\hline File Information & PartB for Huscap.pdf \\
\hline
\end{tabular}

Instructions for use 


\title{
Estimating the value of travel time and of travel time reliability in road networks
}

\author{
Kenetsu Uchida* \\ Graduate School of Engineering, Hokkaido University, Japan \\ North 13 West 8 Kita-ku Sapporo, Japan, 060-8628 \\ Email: uchida@eng.hokudai.ac.jp \\ Tel.: +81-11-706-6210 \\ *corresponding author
}

\begin{abstract}
This study proposes two network models which simultaneously estimate the value of travel time and of travel time reliability based on the risk-averse driver's route choice behaviour. The first model is formulated as a utility maximization problem under monotonic and separable link travel times, whereas the second model is formulated as a utility maximization problem under non-monotonic and non-separable link travel times. The proposed models have the same structure as a user equilibrium (UE) traffic assignment problem with elastic demand. It is shown that the first model, which addresses independent stochastic capacity, is formulated as an optimization problem with a unique solution and is solved by using an algorithm for a UE traffic assignment problem with fixed demand. The second model, which addresses both stochastic Origin-Destination (O-D) flow and stochastic link capacity, is formulated as a nonlinear complementary problem. O-D demand functions formulated in the proposed models are derived from the utility maximization behaviour of the driver in the network. Therefore, the network models proposed in this study are consistent with those of studies that address the value of travel time and of travel time reliability based on utility maximization behaviour without considering the driver's route choice. Numerical experiments are carried out to demonstrate the models presented in this study.
\end{abstract}

Keywords: value of travel time, value of travel time reliability, road network

\section{INTRODUCTION}

In light of the need to evaluate travel time reliability in terms of its effect on mobility in transport networks, many studies have developed models that address uncertainties in the network. Uncertainties in the network can be categorized into the three main factors of supply, demand and travel behaviour. Studies on travel time reliability in the network began with those that address stochastic OriginDestination (O-D) demand flow. By assuming O-D demand flow that follows normal distributions, Asakura and Kashiwadani (1991) solved User Equilibrium (UE) traffic assignment problems several times by using a set of O-D demand flows that were sampled from normal distributions for the purpose of estimating travel time reliability. Clark and Watling (2005) proposed an equilibrium model that calculates travel time reliability under stochastic O-D demand flow when that flow follows a Poisson distribution. They employed probit-based Stochastic User Equilibrium (SUE) for the driver's route choice behaviour. They applied a method proposed by Isserlis (1918) for the purpose of calculating travel time reliability, i.e., variance of stochastic travel time. Nakayama and Takayama (2003) proposed an equilibrium model which assumes that O-D demand flow follows a binomial distribution. UE traffic assignment was employed for expressing the driver's route choice behaviour. They calculated travel time reliability by applying a moment-generating function. Zhou and Chen (2008) proposed an equilibrium model which assumes that O-D demand flow follows a lognormal distribution. The driver's route choice behaviour based on UE was employed. Shao et al. (2006) proposed an equilibrium model which assumed an O-D demand flow that followed a normal distribution. The 
driver's route choice behaviour based on logit-based SUE was employed. They evaluated travel time reliability from the viewpoint of safety margin.

Many studies have addressed stochastic link capacity. Cascetta (1989) and Cascetta and Canterella (1991) developed dynamic traffic assignment models by using a Markov chain. The models developed in their studies calculated drivers' equilibrium states under stochastic link capacity. Bell et al. (1993) proposed a method that estimated travel time reliability under stochastic link capacity by applying sensitivity analysis for logit-based SUE. Cassir and Bell (2002) applied the same method as proposed in Bell et al. (1993) and calculated the travel time reliability by assuming both stochastic O-D demand flow and stochastic link capacity. Chen et al. (1999) analyzed network capacity reliability under stochastic link capacity. They applied the Monte Carlo simulation technique for calculating the network capacity reliability. Chen et al. (2002) analyzed network capacity reliability under both stochastic link capacity and stochastic O-D demand flow by applying the Monte Carlo simulation technique. Lo and Tung (2003) proposed an equilibrium model in which link capacity was assumed to follow a uniform distribution. Stochastic link travel time under stochastic link capacity was calculated by applying a Mellin transform. Uchida and Munehiro (2010) proposed a method that estimated stochastic link capacity from observed traffic data, i.e., density and velocity. They also proposed an equilibrium network model under stochastic link capacity that followed an independent normal distribution. For the purpose of calculating travel time reliability, they applied a method proposed by Bras and Georgakakos (1989) which approximated the first negative moment of a normal distribution by a linear function of the normal distribution and its mean.

It is natural to consider both stochastic O-D demand flow and stochastic link capacity in calculating travel time reliability. Lam et al (2008) proposed an equilibrium model under stochastic O-D demand flow that considered the influence of adverse weather on link capacity. They assumed that the rate of decrease in link capacity followed a normal distribution. Shao et al. (2008) extended the model proposed by Lam et al. (2008) to a model under a multi-user-class network. Sumalee et al. (2011) extended the same model to a model under a multi-modal network.

Watling (2006) proposed an equilibrium model under stochastic travel time that followed a normal distribution. The driver's route choice behaviour was expressed by using a probit-based SUE that also considered arrival penalty. $\mathrm{Wu}$ and Nie (2011) proposed a unified approach to modelling heterogonous risk-taking behaviour in route choice based on the theory of stochastic dominance (SD). They analyzed the relationship between an SD-based approach and other route choice models that considered risktaking behaviour. In these studies, travel time was expressed by a stochastic variable without identifying the source of uncertainties in a network. These studies focused on the driver's risk-taking behaviour. $\mathrm{Ng}$ et al. (2011) proposed a methodology to assess travel time reliability under unknown travel time distribution. The method proposed in their study is useful when we do not have enough data to calibrate the travel time distribution. Chen et al. (2011) extended the $\alpha$-reliable mean-excess traffic equilibrium (METE) model proposed in Chen and Zhou (2010) by explicitly modelling the stochastic perception errors within the travellers' route choice decision processes. $\mathrm{Ng}$ and Waller (2010) presented a methodology based on the theory of Fourier transforms to assess travel time reliability in a transportation network, when the source of uncertainty is given by random road capacities.

For the purpose of measuring the benefits from traffic measures in road networks in terms of travel time and travel time reliability, the value of travel time and of travel time reliability need to be estimated. These can be estimated by using empirical models (e.g., Lam and Small (2001), Brownstone and Small (2005)). Hensher et al. (2011) modified a random utility model to include attribute-specific extended expected utility forms incorporating decision weights and risk in the context of willingness to pay for travel time variability for car commuting travel. Börjesson et al. (2012) estimated the value of travel time variability by applying both a scheduling model and an implied reduced-form model, using stated-choice data. Studies that addressed the value of travel time based on a utility maximization problem without considering the traveller's route choice behaviour in the network began with Becker (1965) and DeSerpa (1971). Recently, some analytical models that 
address the value of travel time and of travel time reliability based on utility maximization principle have been proposed. Fosgerau and Karlström (2010) presented a model that estimates the value of travel time variability based on scheduling preferences. Fosgerau and Engelson (2011) considered the value of travel time variability under scheduling preferences that were defined in terms of linearly time-varying utility rates associated with being at the origin and at the destination. They have shown that a measure related to travel time variability is variance of travel time.

Studies that address travel time reliability based on equilibrium network models have not discussed how to estimate the value of travel time and of travel time reliability. The value of travel time and of travel time reliability were given assumed values in the studies. On the other hand, studies that addressed the value of travel time and of travel time reliability as a utility maximization problem have not addressed the traveller's route choice behaviour in the network, even though this is an essential factor in estimating these values. The present study proposes two network models that estimate the value of travel time and of travel time reliability. The models are derived from a utility maximization problem under budget constraints. The models are finally formulated as UE traffic assignment problems with elastic demand that express the risk-averse driver's route choice. By applying the network models proposed in this study, the value of travel time and of travel time reliability consistent with observed link flows can be estimated. One advantage of the models proposed in this study over utility-maximization-based models is that the value of travel time and of travel time reliability are estimated by taking account of the risk-averse driver's route choice behaviour in a road network that is not considered in the utility-maximization-based models. However, the value of travel time and of travel time reliability determined by the models proposed in this study need to be numerically estimated, whereas the values determined by utility-maximization-based models can be analytically estimated. This point means that sensitivity analysis based on the utility-maximization-based model is easier than sensitivity analysis based on the models proposed in this study.

\section{LINK FLOW AND LINK CAPACITY: RANDOM VARIABLES}

\subsection{Notations}

The notations below are adopted for expressing traffic flow characteristics in a road network in this study.

$\begin{array}{ll}\mathrm{N} & \text { Set of nodes in the network } \\ \mathrm{A} & \text { Set of links in the network } \\ \mathrm{I} & \text { Set of O-D pairs in the network } \\ \mathrm{J}_{i} & \text { Set of routes between O-D pair } i \\ \delta_{a j} & \text { Variable that equals } 1 \text { if link } a \text { is part of route } j, \text { and } 0 \text { otherwise } \\ Q_{i} & \text { Stochastic traffic demand for O-D pair } i \\ q_{i} & \text { Deterministic traffic demand for O-D pair } i \\ F_{i j} & \text { Stochastic flow on route } j \text { between O-D pair } i \\ f_{i j} & \text { Deterministic flow on route } j \text { between O-D pair } i \\ V_{a} & \text { Stochastic flow on link } a \\ v_{a} & \text { Deterministic flow on link } a \\ p_{i j} & \text { Route choice probability of traffic demand for O-D pair } i \text { when route } j \text { is chosen } \\ C_{a} & \text { Stochastic capacity of link } a \\ t_{a}(\cdot) & \text { Travel time function of link } a \\ \rho_{a}(.) & \text { Travel cost function of link } a \\ \sigma_{a}^{2}(.) & \text { Variance function of stochastic link travel time of } a\end{array}$


$\sigma_{a b}(\cdot, \cdot) \quad$ Covariance function of stochastic link travel times of $a$ and $b\left(\sigma_{a}^{2}(\cdot)=\sigma_{a b}(\cdot, \cdot)\right.$ if $\left.a=b\right)$.

$\overline{c v}_{i} \quad$ Coefficient of variation of $Q_{i}$

$c v_{a} \quad$ Coefficient of variation of $V_{a}$

$r_{a b} \quad$ Coefficient of correlation between $V_{a}$ and $V_{b}$

$\breve{r}_{a b} \quad$ Coefficient of correlation between $t_{a}(\cdot)$ and $t_{b}($.

\subsection{Link Flow and Link Capacity}

It may be reasonable to assume that stochastic demand flow, $Q_{i}$, follows an independent theoretical distribution, such as a normal, lognormal, Poisson, binomial or other distribution (Clark and Watling, 2005; Nakayama and Takayama, 2003; Zhou and Chen, 2008; Shao et al., 2006, and so on), that is characterized only by mean and variance. The stochastic demand flow follows an independent random variable with a mean of $E\left[Q_{i}\right]$ and a variance of $\operatorname{var}\left[Q_{i}\right]$. It is reasonable to assume that

$$
\operatorname{var}\left[Q_{i}\right]=\left(\overline{c v}_{i} \cdot E\left[Q_{i}\right]\right)^{2}
$$

where $\overline{c v}_{i}$ is the coefficient of variation of $Q_{i}$. By applying the assumption that the coefficient of variation of path flow is equal to that of O-D demand as employed in Chen et al. (2011), stochastic flow on route $j \in \mathrm{J}_{i}, F_{i j}$, is then given by

$$
F_{i j}=p_{i j} \cdot Q_{i} \forall i \in \mathrm{I}, \forall j \in \mathrm{J}_{\mathrm{i}}
$$

$F_{i j}$ is the random variable with a mean of $E\left[F_{i j}\right]=p_{i j} \cdot E\left[Q_{i}\right]$ and a covariance of $\operatorname{cov}\left[F_{i j}, F_{i k}\right\rfloor=p_{i j} \cdot p_{i k} \cdot \operatorname{var}\left[Q_{i}\right]$, where $p_{i j}\left(j \in \mathrm{J}_{i}\right)$ is route choice probability, which can be determined by a route choice model, e.g., the UE traffic assignment model, the SUE traffic assignment model and so on.

Stochastic flow on link $a, V_{a}$, is given by

$$
V_{a}=\sum_{i \in \mathrm{I}} \sum_{j \in \mathrm{J}_{i}} \delta_{a j} \cdot F_{i j} \quad \forall a \in \mathrm{A}
$$

Therefore, the link flow is a random variable with a mean of

and a covariance of

$$
E\left[V_{a}\right]=\sum_{i \in \mathrm{I}} \sum_{j \in \mathrm{J}_{i}} \delta_{a j} \cdot E\left[F_{i j}\right]=\sum_{i \in \mathrm{I}} \sum_{j \in \mathrm{J}_{i}} \delta_{a j} \cdot p_{i j} \cdot E\left[Q_{i}\right] \forall a \in \mathrm{A}
$$

$$
\operatorname{cov}\left[V_{a}, V_{b}\right]=\sum_{i \in \mathrm{I}} \sum_{j \in \mathrm{J}_{i}} \delta_{a j} \cdot \delta_{b j} \cdot \operatorname{var}\left[F_{i j}\right]=\sum_{i \in \mathrm{I}} \sum_{j \in \mathrm{J}_{i}} \delta_{a j} \cdot \delta_{b j} \cdot\left(p_{i j} \cdot \overline{c v}_{i} \cdot E\left[Q_{i}\right]\right)^{2} \forall a, b \in \mathrm{A}
$$

By introducing $r_{a b}$ and $c v_{a}$, which are respectively the coefficient of correlation between $V_{a}$ and $V_{b}$ $(\forall a, b \in \mathrm{A})$ and the coefficient of variation of $V_{a}(\forall a \in \mathrm{A})$, given by

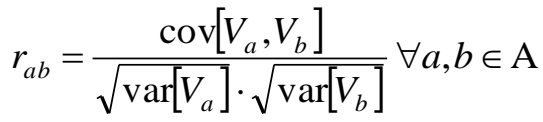

$$
\begin{aligned}
& c v_{a}=\frac{\sqrt{\operatorname{var}\left[V_{a}\right]}}{E\left[V_{a}\right]} \forall a \in \mathrm{A}
\end{aligned}
$$

we obtain the different expression of $\operatorname{cov}\left[V_{a}, V_{b}\right]$ using $E\left[V_{a}\right]$ and $E\left[V_{b}\right]$ given by

$$
\operatorname{cov}\left[V_{a}, V_{b}\right]=r_{a b} \cdot c v_{a} \cdot c v_{b} \cdot E\left[V_{a}\right] \cdot E\left[V_{b}\right] \forall a, b \in \mathrm{A}
$$

It may be reasonable to assume that stochastic link capacity, $C_{a}(a \in \mathrm{A})$, follows a theoretical 
distribution, e.g., a normal distribution (Cassir and Bell, 2002; Lam et al., 2008, Sumalee et al., 2011; Shao et al., 2008; Uchida and Munehiro, 2010), that is characterized only by mean and variance, and that the stochastic link capacity is distributed independently of the O-D flow. Then, the stochastic capacity follows a random variable with a mean of $E\left[C_{a}\right](a \in \mathrm{A})$ and with a variance and covariance of $\operatorname{var}\left[C_{a}\right]$ and $\operatorname{cov}\left[C_{a}, C_{b}\right](a, b \in \mathrm{A})$, respectively.

\section{DRIVER'S ROUTE CHOICE}

\subsection{Travel Time}

Stochastic link travel time can be expressed by the function of $V_{a}$ with given $C_{a}$, i.e., $t_{a}\left(V_{a} ; C_{a}\right)$ by applying the conventional Bureau of Public Roads (BPR) function. The stochastic travel time of route $j$ which serves O-D pair $i, \breve{\Xi}_{i j}$, is given by

$$
\bar{\Xi}_{i j}=\sum_{a \in \mathrm{A}} t_{a}\left(V_{a} ; C_{a}\right) \cdot \delta_{a j} \forall i \in \mathrm{I}, \forall j \in \mathrm{J}_{i} .
$$

The mean and variance of the stochastic route travel time are respectively given by

$$
\begin{gathered}
E\left[\breve{\Xi}_{i j}\right]=\sum_{a \in \mathrm{A}} E\left[t_{a}\left(V_{a} ; C_{a}\right)\right] \cdot \delta_{a j} \forall i \in \mathrm{I}, \forall j \in \mathrm{J}_{i} \\
\operatorname{var}\left[\breve{\Xi}_{i j}\right]=\sum_{a \in \mathrm{A}} \operatorname{var}\left[t_{a}\left(V_{a} ; C_{a}\right)\right] \cdot \delta_{a j}+2 \cdot \sum_{a \in \mathrm{A}} \sum_{b \neq a} \operatorname{cov}\left[t_{a}\left(V_{a} ; C_{a}\right), t_{b}\left(V_{b} ; C_{b}\right)\right] \cdot \delta_{a j} \cdot \delta_{b j} \\
=\sum_{a \in \mathrm{A}} \sigma_{a}^{2}\left(V_{a} ; C_{a}\right) \cdot \delta_{a j}+2 \cdot \sum_{a \in \mathrm{A}} \sum_{b \neq a} \sigma_{a b}\left(V_{a}, V_{b} ; C_{a}, C_{b}\right) \cdot \delta_{a j} \cdot \delta_{b j} \\
=\sum_{a \in \mathrm{A}} \sum_{b \in \mathrm{A}} \sigma_{a b}\left(V_{a}, V_{b} ; C_{a}, C_{b}\right) \cdot \delta_{a j} \cdot \delta_{b j} \forall i \in \mathrm{I}, \forall j \in \mathrm{J}_{i}
\end{gathered}
$$

(1) and (2) can be calculated by applying a method proposed by Isserlis (1918) under a normal distributional assumption. One can refer to Clark and Watling (2005) for the calculations under the assumption that $V_{a}(\forall a \in \mathrm{A})$ follows a multivariate normal distribution, and to Uchida and Munehiro (2010) for calculations under the assumption that $C_{a}(\forall a)$ follows a multivariate normal distribution. By combining the methods proposed by Clark and Watling (2005) and by Uchida and Munehiro (2010), we can derive (1) and (2) under the stochastic link flow and the stochastic link capacity that follow normal distributions.

By following Clark and Watling (2005) and Uchida and Munehiro (2010), and applying the twodimensional Taylor-series expansion to $t_{a}\left(V_{a} ; C_{a}\right)$ at $E\left[V_{a}\right]$ and $E\left[C_{a}\right]$, the mean link travel time, $E\left[t_{a}\left(V_{a} ; C_{a}\right)\right]$, can be approximated by a function of the multivariate normal moments under the normal assumption. The normal moments for the link flow can be expressed by using $E\left[V_{a}\right]$, since the link flow is also characterized only by the mean, $E\left[V_{a}\right]$, and the variance, $\operatorname{var}\left[V_{a}\right]=\left(c v_{a} \cdot E\left[V_{a}\right]\right)^{2}$. The normal moments for the link capacity can be expressed by $E\left[C_{a}\right]$ and $\operatorname{var}\left[C_{a}\right]$, which are constants. Therefore, we assume that $E\left[t_{a}\left(V_{a} ; C_{a}\right)\right]$ is expressed by a function of $E\left[V_{a}\right]$ and that $E\left[t_{a}\left(V_{a} ; C_{a}\right)\right]$ is integrable with respect to $E\left[V_{a}\right] \forall a \in \mathrm{A}$. Hereinafter we denote $E\left[t_{a}\left(V_{a} ; C_{a}\right)\right]$ simply as $\vec{t}_{a}\left(E\left[V_{a}\right]\right)$. Note that, as discussed in Sumalee and $\mathrm{Xu}$ (2011), this notational system does not mean that $t_{a}\left(E\left[V_{a}\right] ; E\left[C_{a}\right]\right)=E\left[t_{a}\left(V_{a} ; C_{a}\right)\right]$. By applying the Taylor-series expansion to $t_{a}\left(V_{a} ; C_{a}\right)$ and to $t_{a}\left(V_{b} ; C_{b}\right)$, and by applying the same discussions made above, it is reasonable to assume that

$$
\sigma_{a b}\left(V_{a}, V_{b} ; C_{a}, C_{b}\right)=E\left[t_{a}\left(V_{a} ; C_{a}\right) \cdot t_{a}\left(V_{b} ; C_{b}\right)\right]-\vec{t}_{a}\left(E\left[V_{a}\right]\right) \cdot \vec{t}_{b}\left(E\left[V_{b}\right]\right)
$$

is expressed by a function of $E\left[V_{a}\right]$ and $E\left[V_{b}\right]$ with the given coefficient of correlation, $r_{a b}$, and the coefficients of variation, $c v_{a}$ and $c v_{b}$; and that $\sigma_{a b}\left(V_{a}, V_{b} ; C_{a}, C_{b}\right)$ is integrable with respect to both $E\left[V_{a}\right]$ and $E\left[V_{b}\right] \forall a, b \in \mathrm{A}$. We will denote $\sigma_{a b}\left(V_{a}, V_{b} ; C_{a}, C_{b}\right)$ simply as $\vec{\sigma}_{a b}\left(E\left[V_{a}\right], E\left[V_{b}\right]\right)$ in the 
rest of the paper. Approximations by using a Tailor series expansion are provided in Appendix A. As shown in section 5, we have a different expression for the covariance of two link travel times, which is $\vec{\sigma}_{a b}\left(E\left[V_{a}\right], E\left[V_{b}\right]\right)=\breve{r}_{a b} \cdot \vec{\sigma}_{a}\left(E\left[V_{a}\right]\right) \cdot \vec{\sigma}_{b}\left(E\left[V_{b}\right]\right)$, where $\breve{r}_{a b}$ is the coefficient of correlation between $t_{a}(\cdot)$ and $t_{b}($.$) . The same conventions as applied to \vec{t}_{a}\left(E\left[V_{a}\right]\right)$ and $\vec{\sigma}_{a b}\left(E\left[V_{a}\right], E\left[V_{b}\right]\right)$ will be adopted when we address the deterministic link flows, $v_{a}$, instead of the stochastic link flows, $E\left[V_{a}\right]$, i.e., $\vec{t}_{a}\left(v_{a}\right)=E\left[t_{a}\left(v_{a} ; C_{a}\right)\right]$ and $\vec{\sigma}_{a b}\left(v_{a}, v_{b}\right)=\sigma_{a b}\left(v_{a}, v_{b} ; C_{a}, C_{b}\right)$.

\subsection{Travel Cost}

For the purpose of evaluating mean travel time and travel time reliability in terms of their monetary value, we introduce travel cost. Factors that influence travel cost can be fuel consumption and maintenance costs of a vehicle. We assume that the stochastic link travel cost is expressed by the function of $V_{a}$ with a given $C_{a}$, i.e., $\rho_{a}\left(V_{a} ; C_{a}\right)$. The stochastic travel cost on route $j \in \mathrm{J}$ which serves O-D pair $i, \breve{\Gamma}_{i j}$, is given by

$$
\breve{\Gamma}_{i j}=\sum_{a \in \mathrm{A}} \rho_{a}\left(V_{a} ; C_{a}\right) \cdot \delta_{a j} \forall i \in \mathrm{I}, \forall j \in \mathrm{J}_{i}
$$

The mean of the route travel cost is given by

$$
E\left[\breve{\Gamma}_{i j}\right]=\sum_{a \in \mathrm{A}} E\left[\rho_{a}\left(V_{a} ; C_{a}\right)\right] \cdot \delta_{a j} \forall i \in \mathrm{I}, \forall j \in \mathrm{J}_{i}
$$

We assume that $E\left[\rho_{a}\left(V_{a} ; C_{a}\right)\right]$ is expressed by a function of $E\left[V_{a}\right]$ and that $E\left[\rho_{a}\left(V_{a} ; C_{a}\right)\right]$ is integrable with respect to $E\left[V_{a}\right] \forall a \in \mathrm{A}$. Therefore, we hereinafter denote $E\left[\rho_{a}\left(V_{a} ; C_{a}\right)\right]$ simply as $\vec{\rho}_{a}\left(E\left[V_{a}\right]\right)$. The same convention as applied to $\vec{t}_{a}\left(E\left[V_{a}\right]\right)$ is adopted when we address the deterministic link flows, $v_{a}$, instead of the stochastic links flows, $E\left[V_{a}\right]$, i.e., $\vec{\rho}_{a}\left(v_{a}\right)=E\left[\rho_{a}\left(v_{a} ; C_{a}\right)\right]$. The same discussions made in the previous section have been applied in this section.

\subsection{Route Choice Problem}

Mean travel time, mean travel cost and variance of travel time were formulated in the previous section. The risk-averse driver may take into account the variation of route travel time, such as the variance of route travel time, as well as the mean travel time and the mean travel cost in their route choice decisions. Following Fosgerau and Engelson (2011), we define the travel time reliability in this study as the variance of the stochastic travel time. Following Lo and Chen (2000), by using parameters, $\lambda>0, \gamma>0$ and $\omega>0$, the risk-averse driver's route choice problem can be formulated as the following nonlinear complementary problem:

where

$$
\text { find } \mathbf{z}=\left(\mathbf{F}^{*} \mathbf{d}^{*}\right)^{T} \text { such that } \hat{\mathbf{z}} \geq \mathbf{0}, \mathbf{h}(\hat{\mathbf{z}}) \geq \mathbf{0}, \hat{\mathbf{z}}^{T} \mathbf{h}(\hat{\mathbf{z}})=0
$$

$$
\begin{aligned}
& \mathbf{F}=\left(\begin{array}{lllllll}
F_{11} & \ldots & F_{1\left|\mathrm{~J}_{1}\right|} & \ldots & F_{\mid[\mid \mathrm{I}} & \ldots & F_{\mathrm{I}|\mathrm{J} / \mathrm{I}|}
\end{array}\right)^{T} \\
& \mathbf{d}=\left(\begin{array}{lll}
d_{1} & \ldots & d_{\mid[\mid}
\end{array}\right)^{T} \\
& \hat{\mathbf{z}}=(E[\mathbf{F}] \mathbf{d})^{T} \\
& \mathbf{h}(\hat{\mathbf{z}})=\left(\begin{array}{ll}
\mathbf{g} & \mathbf{q}
\end{array}\right)^{T}
\end{aligned}
$$

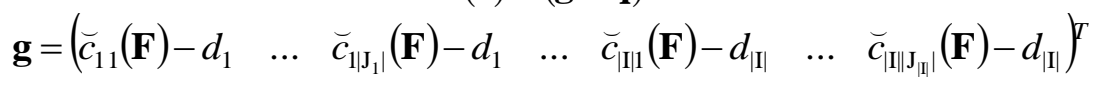

$$
\begin{aligned}
& \mathbf{q}=\left(\sum_{j \in \mathrm{J}_{i}} E\left[F_{1 j}\right]-E\left[Q_{i}\right] \quad \ldots \quad \sum_{j \in \mathrm{J}_{[||}} E\left[F_{|I| j}\right]-E\left[Q_{|I|}\right]\right)^{T}
\end{aligned}
$$




$$
\breve{c}_{i j}(\mathbf{F})=\lambda \cdot E\left[\breve{\Xi}_{i j}\right]+\omega \cdot E\left[\breve{\Gamma}_{i j}\right]+\gamma \cdot \operatorname{var}\left[\breve{\Xi}_{i j}\right]
$$

$\mathbf{F}^{*}$ and $\mathbf{d}^{*}$ are the vector of equilibrium stochastic route flows and the vector of minimum route performance values for all O-D pairs by which the driver's route choice is determined. The above formulation means that if a route is used by the driver, the route performance value, $\breve{c}_{i j}(\mathbf{F})$, is equal to the minimum route performance value, $d_{i}^{*}$, which is smaller than or equal to the performance values of unused routes. In the rest of the paper, a variable, a parameter or a vector of variables to which the superscript $*$ is put means an optimized or equilibrated one.

The above problem can be solved by minimizing the following gap function (Lo and Chen, 2000; Watling, 2006):

where

$$
\min \operatorname{gap}(\mathbf{z})=\sum_{k} \varphi\left(\hat{z}_{k}, h_{k}(\hat{\mathbf{z}})\right)
$$

$$
\varphi(a, b)=\frac{1}{2} \cdot\left(\sqrt{a^{2}+b^{2}}-(a+b)\right)^{2}
$$

\section{VALUES OF TRAVEL TIME AND TRAVEL TIME RELIABILITY}

\subsection{Monotonic and Separable Case}

In the previous section, both O-D demand flow and link capacity were assumed to follow random variables. In this section, we consider a situation where the link capacity is represented by an independent stochastic variable whereas the O-D demand flow is represented by a deterministic variable that will be denoted by $q_{i}$ instead of $Q_{i}$. This assumption may be unrealistic, since it is generally believed that travel time reliability is path-based. However, the formulation presented in this section will play an important role in driving the path-based formulation to be presented in the next section. Without a loss of generality, $q_{i}$ can be regarded as $q_{i}=E\left[Q_{i}\right]$. Thus, the deterministic route flow is then

$$
f_{i j}=p_{i j} \cdot q_{i} \forall i \in \mathrm{I}, \forall j \in \mathrm{J}_{i}
$$

The link capacity is still denoted by $C_{a}(a \in \mathrm{A})$ with the mean of $E\left[C_{a}\right](a \in \mathrm{A})$ and the variance and covariance of $\operatorname{var}\left[C_{a}\right]$ and $\operatorname{cov}\left[C_{a}, C_{b}\right]=0$, respectively. By using the deterministic link flow, $v_{a}$, stochastic route travel time is then

$$
\Xi_{i j}=\sum_{a \in \mathrm{A}} t_{a}\left(v_{a} ; C_{a}\right) \cdot \delta_{a j} \forall i \in \mathrm{I}, \forall j \in \mathrm{J}_{i}
$$

where

$$
v_{a}=\sum_{i \in \mathrm{I}} \sum_{j \in \mathrm{J}_{i}} f_{i j} \cdot \delta_{a j} \forall a \in \mathrm{A}
$$

In a similar way, the stochastic route travel cost is

$$
\Gamma_{i j}=\sum_{a \in \mathrm{A}} \rho_{a}\left(v_{a} ; C_{a}\right) \cdot \delta_{a j} \forall i \in \mathrm{I}, \forall j \in \mathrm{J}_{i}
$$

The mean values of route travel time and route travel cost are respectively given by

$$
E\left[\Xi_{i j}\right]=\sum_{a \in \mathrm{A}} \vec{t}_{a}\left(v_{a}\right) \cdot \delta_{a j} \forall i \in \mathrm{I}, \forall j \in \mathrm{J}_{i}
$$




$$
E\left[\Gamma_{i j}\right]=\sum_{a \in \mathrm{A}} \vec{\rho}_{a}\left(v_{a}\right) \cdot \delta_{a j} \forall i \in \mathrm{I}, \forall j \in \mathrm{J}_{i}
$$

In contrast, the variance of the route travel time is

$$
\operatorname{var}\left[\Xi_{i j}\right]=\sum_{a \in \mathrm{A}} \vec{\sigma}_{a}^{2}\left(v_{a}\right) \cdot \delta_{a j} \forall i \in \mathrm{I}, \forall j \in \mathrm{J}_{i}
$$

Note that, since we assume $\operatorname{cov}\left[C_{a}, C_{b}\right]=0(a \neq b \in \mathrm{A})$, therefore we obtain $\vec{\sigma}_{a b}\left(v_{a}, v_{b}\right)=0$ (Uchida and Munehiro, 2010). We assume for the time being that $\vec{t}_{a}\left(v_{a}\right), \vec{\rho}_{a}\left(v_{a}\right)$ and $\vec{\sigma}_{a}^{2}\left(v_{a}\right)$ are all monotonic increasing functions of $v_{a}$.

\subsection{Utility Maximization under the Monotonic and Separable Case}

In this section, a model that estimates the value of (mean) travel time and of travel time reliability is formulated. We set the following three assumptions for the estimation.

A1. Link flows, route flows and O-D flows in the network can be observed or estimated. Considering the evolution of ITS technology, it may be possible to observe a large number of link flows in a road network. By applying an O-D flow estimation technique using a partial set of observed link flows, e.g., Sherali et al. (2003), O-D flows and route flows as well as link flows in the network can be estimated. The observed or estimated flow can be regarded as mean flow.

A2. Observed or estimated traffic flows are generated from the optimal behaviour of each driver in the network such that the utility level of each driver is maximized subject to the three budget constraints of mean travel time, mean travel cost and travel time reliability. We applied the same assumption as employed in utility theory: that the observed behaviour is generated based on the utility maximization principle.

A3. Three budget constraints in A2 can be calculated by using the observed or estimated traffic flows. If we know the link performance functions in the network that is to be formulated later, it will be shown that the three budget constraints can be calculated from the observed or estimated traffic flows. The link performance function can be estimated by calibrating parameters in the function from link performance data.

In the rest of paper, observed or estimated link flows are shown by $\hat{v}_{a}$. We employ this convention for all kinds of traffic flows. For the purpose of expressing the driver's route choice behaviour based on marginal private costs of mean travel time, mean travel cost and travel time reliability, we will introduce three variables:

$$
\begin{gathered}
\tilde{t}_{a}\left(v_{a}\right)= \begin{cases}\int_{0}^{v_{a}} \vec{t}_{a}(w) \cdot d w & \text { if } v_{a}>0 \\
v_{a} & \text { otherwise }\end{cases} \\
\tilde{\rho}_{a}\left(v_{a}\right)=\left\{\begin{array}{cc}
\int_{0}^{v_{a}} \vec{\rho}_{a}(w) \cdot d w & \text { if } v_{a}>0 \\
\frac{v_{a}}{0} & \text { otherwise }
\end{array}\right. \\
\tilde{\sigma}_{a}^{2}\left(v_{a}\right)=\left\{\begin{array}{cc}
\int_{0}^{v_{a}} \vec{\sigma}_{a}^{2}\left(w_{a}\right) \cdot d w_{a} & \text { if } v_{a}>0 \\
\frac{v_{a}}{0} & \text { otherwise }
\end{array}\right.
\end{gathered}
$$


(7), (8) and (9) will be used to derive three indexes for mean travel time, mean travel cost and travel time reliability. Then, consider the following primary problem [PP]:

$$
v=\max u\left(q_{1}, \ldots, q_{|I|}\right)=\sum_{i \in \mathrm{I}} \int_{0}^{q_{i}} \frac{\alpha_{i}}{w+1} \cdot d w
$$

s.t.

$$
\begin{gathered}
\sum_{i \in \mathrm{I}} t_{i} \cdot q_{i} \leq \phi \\
\sum_{i \in \mathrm{I}} \rho_{i} \cdot q_{i} \leq \pi \\
\sum_{i \in \mathrm{I}} \sigma_{i}^{2} \cdot q_{i} \leq \theta \\
q_{i}+e_{i}=\breve{q}_{i} \forall i \in \mathrm{I}
\end{gathered}
$$

and (5) and (6), where

$$
\begin{gathered}
t_{i}=\sum_{j \in \mathrm{J}_{i}} \sum_{a \in \mathrm{A}} p_{i j} \cdot \tilde{t}_{a}\left(v_{a}\right) \cdot \delta_{a j}=\sum_{j \in \mathrm{J}_{i}} \sum_{a \in \mathrm{A}} \frac{f_{i j}}{q_{i}} \cdot \tilde{t}_{a}\left(v_{a}\right) \cdot \delta_{a j} \forall i \in \mathrm{I} \\
\rho_{i}=\sum_{j \in \mathrm{J}_{i}} \sum_{a \in \mathrm{A}} p_{i j} \cdot \tilde{\rho}_{a}\left(v_{a}\right) \cdot \delta_{a j}=\sum_{j \in \mathrm{J}_{i}} \sum_{a \in \mathrm{A}} \frac{f_{i j}}{q_{i}} \cdot \tilde{\rho}_{a}\left(v_{a}\right) \cdot \delta_{a j} \forall i \in \mathrm{I} \\
\sigma_{i}^{2}=\sum_{j \in \mathrm{J}_{i}} \sum_{a \in \mathrm{A}} p_{i j} \cdot \tilde{\sigma}_{a}^{2}\left(v_{a}\right) \cdot \delta_{a j}=\sum_{j \in \mathrm{J}_{i}} \sum_{a \in \mathrm{A}} \frac{f_{i j}}{q_{i}} \cdot \tilde{\sigma}_{a}^{2}\left(v_{a}\right) \cdot \delta_{a j} \forall i \in \mathrm{I} \\
\phi=\sum_{a \in \mathrm{A}} \tilde{t}_{a}\left(\hat{v}_{a}\right) \cdot \hat{v}_{a} \\
\pi=\sum_{a \in \mathrm{A}} \tilde{\rho}_{a}\left(\hat{v}_{a}\right) \cdot \hat{v}_{a} \\
\theta=\sum_{a \in \mathrm{A}} \tilde{\sigma}_{a}^{2}\left(\hat{v}_{a}\right) \cdot \hat{v}_{a}
\end{gathered}
$$

The objective function $u$ in (10) can be regarded as a direct utility function (Varian, 1984) in the network, since the objective function becomes

$$
u\left(q_{1}, \ldots, q_{|I|}\right)=\sum_{i \in \mathrm{I}} \int_{0}^{q_{i}} \frac{\alpha_{i}}{w+1} \cdot d w=\sum_{i \in \mathrm{I}} \alpha_{i} \cdot \ln \left(q_{i}+1\right)
$$

(17) follows the style of a Cobb-Douglas utility function (Cobb and Douglas, 1928) by assuming that the O-D demand flow of $q_{i}$ is the amount of substitute trip $i$, and that $\alpha_{i}(>0)$ is the parameter such that $\sum_{i} \alpha_{i}=1$. The constraints shown by (11)-(13) can be regarded as budget constraints in which three different prices, i.e., $t_{i}, \rho_{i}$ and $\sigma_{i}^{2}$, are offered to the O-D flow $q_{i}$. The utility function (17) means that the more the O-D flows increase, the higher the utility level is. However, these three budget constraints do not allow the O-D flows to increase independently of the observed link flows. In fact, the utility level is maximized at the observed link flows if the parameter $\alpha_{i}$ is calibrated precisely based on a method shown in Appendix B. The utility maximization problem under budget constraints shown by (11)-(13) can be obtained from the individual driver's utility maximization problem (Appendix B). As mentioned earlier, a calibration method of the distribution parameter $\alpha_{i}$ is also discussed in Appendix B. In (17), $\ln \left(q_{i}+1\right)$ can be regarded as a partial utility gain that is obtained by consuming the O-D demand of $q_{i}$. It may be reasonable to regard (17) as an expected utility by assuming $\alpha_{i}$ as a probability. The Cobb-Douglas utility function has a property whereby the amount of goods consumed is determined only by the price of the goods, i.e., the amount of goods consumed is independent of the prices of other goods. This property is consistent with the concept of a traffic assignment model with elastic demand in which an O-D flow is determined only by the performance 
value of the corresponding O-D pair.

Unlike in the standard Cobb-Douglas utility function, $q_{i}+1$ rather than $q_{i}$ is employed for the purpose of equalling the partial utility gain that is obtained by consuming the O-D demand of $q_{i}=0$ to zero. $v$ in (10) represents the corresponding indirect utility function (Varian, 1984). $e_{i}\left(0 \leq e_{i}<\breve{q}_{i}\right)$ in (14) is excess demand (Gartner, 1980) that does not appear in the network. $\breve{q}_{i}$ in (14) is a constant given to each O-D pair $i$ that is larger than the supposed maximal traffic demand. $t_{i}$ is an index for mean travel time between O-D pair $i$. In a similar way, $\rho_{i}$ and $\sigma_{i}^{2}$ are indexes for mean travel cost and travel time reliability between O-D pair $i$, respectively. Following A3, three budget constraints with respect to mean travel time, mean travel cost and travel time reliability, which are respectively denoted by (11)-(13), are calculated by using the observed link flows. Without (5), (6) and (14), PP follows the style of a standard utility maximization problem subject to budget constraints. Different from a standard utility maximization problem under budget constraints, $t_{i}, \rho_{i}$ and $\sigma_{i}^{2}$ can change according to consumption level $\left(q_{i} \forall i\right)$ and to the driver's route choice behaviour.

By applying the relationship between link flows and route flows shown by (6) to (11)-(13), the budget constraints denoted by (11)-(13) can be respectively reformulated as

$$
\begin{aligned}
& \sum_{a \in \mathrm{A}} \int_{0}^{v_{a}} \vec{t}_{a}(w) \cdot d w \leq \phi \\
& \sum_{a \in \mathrm{A}} \int_{0}^{v_{a}} \vec{\rho}_{a}(w) \cdot d w \leq \pi \\
& \sum_{a \in \mathrm{A}} \int_{0}^{v_{a}} \vec{\sigma}_{a}^{2}(w) \cdot d w \leq \theta
\end{aligned}
$$

We solve PP by applying the partial Lagrangian (Lasdon, 1970) with respect to the budget constraints denoted by (18)-(20). Let $\lambda^{*}, \omega^{*}$ and $\gamma^{*}$ denote the optimal multipliers associated with (18), (19) and (20), respectively. The PP is then equivalent to a minimization problem of the Lagrangian $L$ :

$$
\begin{aligned}
-v & =\min L=\lambda^{*} \cdot\left(\sum_{a \in \mathrm{A}} \int_{0}^{v_{a}} \vec{t}_{a}(w) \cdot d w-\phi\right)+\omega^{*} \cdot\left(\sum_{a \in \mathrm{A}} \int_{0}^{v_{a}} \vec{\rho}_{a}(w) \cdot d w-\pi\right) \\
& +\gamma^{*} \cdot\left(\sum_{a \in \mathrm{A}} \int_{0}^{v_{a}} \vec{\sigma}_{a}^{2}(w) \cdot d w-\theta\right)-\sum_{i \in \mathrm{I}} \int_{0}^{q_{i}} \frac{\alpha_{i}}{w+1} \cdot d w,
\end{aligned}
$$

s.t. (5), (6) and (14). By neglecting the constant terms, i.e., $\lambda^{*} \cdot \phi, \omega^{*} \cdot \pi$ and $\gamma^{*} \cdot \theta$, in (21), the above problem can be simplified as

$$
\min \tilde{L}=\sum_{a \in \mathrm{A}} \int_{0}^{v_{a}} \chi_{a}(w) \cdot d w-\sum_{i \in \mathrm{I}} \int_{0}^{q_{i}} \frac{\alpha_{i}}{w+1} \cdot d w
$$

s.t. (5), (6) and (14) where

$$
\chi_{a}(w)=\lambda^{*} \cdot \vec{t}_{a}(w)+\omega^{*} \cdot \vec{\rho}_{a}(w)+\gamma^{*} \cdot \vec{\sigma}_{a}^{2}(w) \forall a \in \mathrm{A}
$$

Since $\chi_{a}\left(v_{a}\right)$ is monotonic increasing function of $v_{a}$ as shown, the above optimization problem has the same structure as a standard UE traffic assignment problem with elastic demand by regarding $\chi_{a}\left(v_{a}\right)$ as link performance function and $\alpha_{i} /\left(q_{i}+1\right)$ as an inverse demand function that is denoted by $D_{i}^{-1}(\mathbf{q})$ where $\mathbf{q}=\left(q_{1}, \ldots, q_{i}, \ldots\right)^{T}$. The second term on the right hand side of (22) is written as 


$$
\begin{aligned}
-\sum_{i \in \mathrm{I}} \int_{0}^{q_{i}} D_{i}^{-1}(\mathbf{q}) \cdot d w & =-\sum_{i \in \mathrm{I}} \int_{0}^{\breve{q}_{i}} \frac{\alpha_{i}}{w+1} \cdot d w+\sum_{i \in \mathrm{I}} \int_{0}^{e_{i}} \frac{\alpha_{i}}{\breve{q}_{i}-w+1} \cdot d w \\
& =\text { const. }-\alpha_{i} \cdot\left(\ln \left(\breve{q}_{i}-e_{i}+1\right)-\ln \left(\breve{q}_{i}+1\right)\right),
\end{aligned}
$$

Therefore, PP is equivalent to the following UE traffic assignment with elastic demand problem [UEED] (Gartner, 1980):

$$
\min \hat{L}=\sum_{a \in \mathrm{A}} \int_{0}^{v_{a}} \chi_{a}(w) \cdot d w+\sum_{i \in \mathrm{I}} \int_{0}^{e_{i}} d_{i}(w) \cdot d w
$$

s.t. (5), (6) and (14), where

$$
d_{i}(w)=\frac{\alpha_{i}}{\breve{q}_{i}-w+1}
$$

We call $\alpha_{i}$ in (23) a prohibitive route performance value for O-D pair $i$, since the O-D demand is calculated as 0 when the corresponding route performance value is $\alpha_{i}$. The value of travel time, $\tau$, which is the marginal rate of substitution of mean travel time for mean travel cost can be given by

$$
\tau=\frac{\partial v / \partial \phi}{\partial v / \partial \pi}=\frac{\lambda^{*}}{\omega^{*}}
$$

In a similar way, the value of travel time reliability, $v$, which is the marginal rate of substitution of travel time reliability for mean travel cost can be given by

$$
v=\frac{\partial v / \partial \theta}{\partial v / \partial \pi}=\frac{\gamma^{*}}{\omega^{*}}
$$

We will discuss first the estimation of the link flows under given optimal multipliers. PP and UE-ED have a unique solution if the Lagrangian $L$ is strictly a concave function with respect to feasible $\lambda, \omega$ and $\gamma$. It is obvious that $-u$ and the three budget constraints shown by (18)-(20) are convex. Therefore, the Lagrangian $L$ is concave with respect to feasible $\lambda, \omega$ and $\gamma$ according to the saddle point theorem. Further, the inequality constraints denoted by (18)-(20) guarantee that $\lambda^{*} \geq 0, \omega^{*} \geq 0$ and $\gamma^{*} \geq 0$. Therefore, $\chi_{a}\left(v_{a}\right) \forall a \in \mathrm{A}$ is monotonic increasing function of $v_{a}$, since we have assumed that $\vec{t}_{a}\left(v_{a}\right), \vec{\rho}_{a}\left(v_{a}\right)$ and $\vec{\sigma}_{a}^{2}\left(v_{a}\right)$ are all monotonic increasing functions of $v_{a}$. Accordingly, if the optimal multipliers $\lambda^{*}, \omega^{*}$ and $\gamma^{*}$ are obtained, UE-ED can be solved without route enumeration by applying an algorithm for the UE traffic assignment problem by adding a dummy link with the link performance function of $d_{i}\left(e_{i}\right)=\alpha_{i} /\left(\breve{q}_{i}-e_{i}+1\right)$ between O-D pair $i$ in the network. This procedure is the same as that in which a dummy link is added to each O-D pair in user equilibrium with elastic demand. Therefore, it can be concluded that PP and UE-ED have a unique solution, since $d_{i}\left(e_{i}\right)$ is a monotonic increasing function of $e_{i}$.

We next discuss the estimation of optimal multipliers. Under a feasible set of $\lambda, \omega$ and $\gamma$, the value of the Lagrangian, $L$, the link flows, $v_{a}(\forall a)$, and the vector of O-D flows, $\mathbf{q}$, can be calculated by applying an algorithm for the UE traffic assignment problem as discussed above. According to the saddle point theorem, the optimal multipliers $\lambda^{*}, \omega^{*}$ and $\gamma^{*}$ maximize the Lagrangian $L$. Therefore, by applying an algorithm that is obtained by combining an algorithm for the UE traffic assignment problem and an algorithm for the convex programming problem, the optimal multipliers can be estimated. In fact, the optimal multipliers are the shadow prices of the budget constraints shown by (18)-(20) with respect to the direct utility function.

The model presented in this section requires model parameters, i.e., the link flow, the link capacity and the calibration parameters for the link performance function to estimate the values of travel time and 
travel time reliability. The utility maximization based model discussed in section 1 also requires the calibration parameters for utility function to estimate these values. However, the number of parameters for the utility maximization based model is in general less than those of the model presented in this section.

It may be useful if the model presented in this paper can simultaneously address markets other than the road transportation market. As discussed in Appendix $\mathrm{C}$, the model proposed in this study can address other markets by applying a nested constant elasticity of substitution (CES) utility function.

\subsection{Utility Maximization under the Non-monotonic and Non-separable Case}

In general, travel cost function is not a monotonic increasing function of link flow, and travel time reliability of a route is not equal to the summation of those of the links that comprise the route due to the covariance terms. The latter case where we have to consider the covariance of link travel times can occur when we address stochastic O-D demand flow and/or correlated stochastic traffic capacity. Therefore, in general, the risk-averse driver's route choice behaviour cannot be formulated as the optimization problem shown in the previous section.

For addressing the non-monotonic and non-separable case, we modify (9) as follows:

$$
\tilde{\sigma}_{a b}\left(E\left[V_{a}\right], E\left[V_{b}\right]\right)=\left\{\begin{array}{cc}
\frac{\int_{0}^{E\left[V_{a}\right] E\left[V_{b}\right]} \int_{0}^{b} \vec{\sigma}_{a b}\left(w_{a}, w_{b}\right) \cdot d w_{b} \cdot d w_{a}}{E\left[V_{a b}\right]} & \text { if } E\left[V_{a b}\right]>0 \\
0 & \text { otherwise }
\end{array}\right.
$$

where

$$
E\left[V_{a b}\right]=\sum_{i \in \mathrm{I}} \sum_{j \in \mathrm{J}_{i}} \sum_{a \in \mathrm{A}} \sum_{b \in \mathrm{A}} E\left[F_{i j}\right] \cdot \delta_{a j} \cdot \delta_{b j}
$$

Also, (15), (16) and (20) need to be modified as follows:

$$
\begin{gathered}
\sigma_{i}^{2}=\sum_{j \in \mathrm{J}_{i}} \sum_{a \in \mathrm{A}} \sum_{b \in \mathrm{A}} p_{i j} \cdot \tilde{\sigma}_{a b}\left(E\left[V_{a}\right], E\left[V_{b}\right]\right) \cdot \delta_{a j} \cdot \delta_{b j}=\sum_{j \in \mathrm{J}_{i}} \sum_{a \in \mathrm{A}} \sum_{b \in \mathrm{A}} \frac{E\left[F_{i j}\right]}{E\left[Q_{i}\right]} \cdot \tilde{\sigma}_{a b}\left(E\left[V_{a}\right], E\left[V_{b}\right]\right) \cdot \delta_{a j} \cdot \delta_{b j} \forall i \in \mathrm{I} \\
\theta=\sum_{a \in \mathrm{A}} \sum_{b \in \mathrm{A}} \tilde{\sigma}_{a b}\left(E\left[\hat{V}_{a}\right], E\left[\hat{V}_{b}\right]\right) \cdot E\left[\hat{V}_{a b}\right] \\
\sum_{a \in \mathrm{A}} \sum_{b \in \mathrm{A}} \int_{0}^{E\left[V_{a} \in E\left[V_{b}\right]\right.} \int_{0}^{\sigma_{a b}} \tilde{\sigma}_{a}\left(w_{a}, w_{b}\right) \cdot d w_{b} \cdot d w_{a} \leq \theta
\end{gathered}
$$

Recall that the route performance value is given by (4). Following Lo and Chen (2000), the equilibrium condition is then

$$
E\left[F_{i j}\right] \cdot\left(\breve{c}_{i j}(\mathbf{F})-d_{i}\left(e_{i}\right)\right)=0, E\left[F_{i j}\right] \geq 0, \breve{c}_{i j}(\mathbf{F})-d_{i}\left(e_{i}\right) \geq 0
$$

The other constraints can be expressed by

$$
\begin{aligned}
\lambda \cdot\left(\phi-\sum_{a \in \mathbf{A}} \int_{0}^{E\left[V_{a}\right]} \vec{t}_{a}(w) \cdot d w\right) & =0, \lambda \geq 0, \phi-\sum_{a \in \mathbf{A}} \int_{0}^{E\left[V_{a}\right]} \vec{t}_{a}(w) \cdot d w \geq 0 \\
\omega \cdot\left(\pi-\sum_{a \in \mathbf{A}} \int_{0}^{E\left[V_{a}\right]} \vec{\rho}_{a}(w) \cdot d w\right) & =0, \omega \geq 0, \pi-\sum_{a \in \mathbf{A}} \int_{0}^{E\left[V_{a}\right]} \vec{\rho}_{a}(w) \cdot d w \geq 0 \\
\gamma \cdot\left(\theta-\sum_{a \in \mathrm{A} b \in \mathrm{A}} \int_{0}^{E\left[V_{a}\right] E\left[V_{b}\right]} \int_{0}^{E\left[V_{a}\right] E\left[V_{b}\right]} \vec{\sigma}_{a b}\left(w_{a}, w_{b}\right) \cdot d w_{a} \cdot d w_{b}\right) & =0, \gamma \geq 0, \theta-\sum_{a \in \mathrm{A} b \in \mathrm{A}} \int_{0} \int_{0} \vec{\sigma}_{a b}\left(w_{a}, w_{b}\right) \cdot d w_{a} \cdot d w_{b} \geq 0 \\
d_{i}\left(e_{i}\right) \cdot\left(\sum_{j \in J_{i}} E\left[F_{i j}\right]+e_{i}-\breve{q}_{i}\right) & =0, d_{i}\left(e_{i}\right) \geq 0, \sum_{j \in J_{i}} E\left[F_{i j}\right]+e_{i}-\breve{q}_{i} \geq 0
\end{aligned}
$$


Therefore, the values of mean travel time and travel time reliability under stochastic O-D demand and correlated stochastic traffic capacity can be estimated by solving the following nonlinear complementary problem [UE-NCP]:

$$
\text { find } \mathbf{z}=\left(\begin{array}{lll}
\mathbf{F}^{*} & \mathbf{p}^{*} \quad \mathbf{e}^{*}
\end{array}\right)^{T} \text { such that } \hat{\mathbf{z}} \geq \mathbf{0}, \mathbf{h}(\hat{\mathbf{z}}) \geq \mathbf{0}, \hat{\mathbf{z}}^{T} \mathbf{h}(\hat{\mathbf{z}})=0
$$

where

$$
\begin{aligned}
& \mathbf{p}=\left(\begin{array}{lll}
\lambda & \omega & \gamma
\end{array}\right)^{T} \\
& \mathbf{e}=\left(\begin{array}{lll}
e_{1} & \ldots & e_{\mid \mathrm{II}}
\end{array}\right)^{T} \\
& \hat{\mathbf{z}}=\left(\begin{array}{lll}
E[\mathbf{F}] & \mathbf{p} & \mathbf{d}(\mathbf{e}))^{T}
\end{array}\right. \\
& \mathbf{d}(\mathbf{e})=\left(\begin{array}{lll}
d_{1}\left(e_{1}\right) & \ldots & d_{|| \mathrm{I}}\left(e_{|| \mathrm{I}}\right)
\end{array}\right)^{T} \\
& \mathbf{h}(\hat{\mathbf{z}})=\left(\begin{array}{lll}
\mathbf{g} & \mathbf{x} & \mathbf{q}
\end{array}\right)^{T}
\end{aligned}
$$

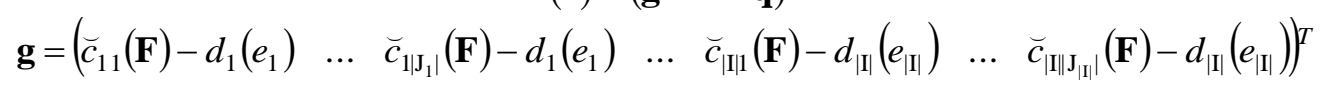

$$
\begin{aligned}
& \mathbf{x}=\left(\phi-\sum_{a \in \mathrm{A}} \int_{0}^{E\left[V_{a}\right]} \vec{t}_{a}\left(v_{a}\right) \cdot d w \quad \pi-\sum_{a \in \mathrm{A}} \int_{0}^{E\left[V_{a}\right]} \vec{\rho}_{a}(w) \cdot d w \quad \theta-\sum_{a \in \mathrm{A}} \sum_{b \in \mathrm{A}} \int_{0}^{E\left[V_{a}\right] E\left[V_{b}\right]} \int_{0}^{T} \vec{\sigma}_{a b}\left(w_{a}, w_{b}\right) \cdot d w_{a} \cdot d w_{b}\right)^{T} \\
& \mathbf{q}=\left(\sum_{j \in \mathrm{J}_{i}} E\left[F_{1 j}\right]+e_{1}-\breve{q}_{1} \quad \cdots \quad \sum_{j \in \mathrm{J}_{|| \mid}} E\left[F_{|I| j}\right]+e_{\mid[\mid}-\breve{q}_{|I|}\right)^{T}
\end{aligned}
$$

and (3), (4).

In the above formulation, a user equilibrium problem with elastic demand is formulated as a nonlinear complementary problem that simultaneously considers not only the constraints on mean travel time, mean travel cost and travel time reliability denoted by (25) but also the corresponding multipliers denoted by (24). UE-ED was extended to UE-NCP so that the path-based travel time reliability could be addressed. There are three differences from the model presented in section 3.3. The first point is that the model in section 3.3 assumes that the three parameters $\lambda, \omega$ and $\gamma$ are given, whereas these parameters are the decision variables in UE-NCP. As mentioned earlier, these decision variables are the shadow prices of the three budget constraints. The second point is that the model in section 3.3 addresses fixed demand, whereas UE-NCP addresses elastic demand. Therefore, the O-D demand is also the decision variable in UE-NCP. In solving UE-NCP, route enumeration is required, since UENCP is formulated as a route-based problem due to the non-separable property of travel time reliability. However, this property is no longer a limitation of the model, since we can apply one of the efficient route enumeration techniques (e.g., Zijpp and Catalano (2005)) to obtain a path set in the network. The final point is that the minimum route performance value is determined by the function $d_{i}\left(e_{i}\right)$, which expresses the relationship between excess demand and route performance. Note that without loss of generality, the formulation shown above can address a problem under the monotonically increasing and separable link performance function. Unlike PP and UE-ED, however, the uniqueness of the solution for UE-NCP is not guaranteed, since a non-monotonic and non-separable link performance function is assumed. For this reason, we cannot formulate the user equilibrium problem as convex programming that has a unique solution.

\section{NUMERICAL EXPERIMENTS}

\subsection{Experiment under Unknown Values of Travel Time and Travel Time Reliability}

This section addresses a problem under the non-separable case. As explained in the previous section, we have to consider covariance of link travel times in this experiment. Therefore, the formulation shown in section 4.3, i.e., UE-NCP, is applied for expressing the risk-averse driver's route choice behaviour. A test network with the O-D demand flow shown in Figure 1 will be addressed in this section. The dotted line in Figure 1 is a dummy link. 


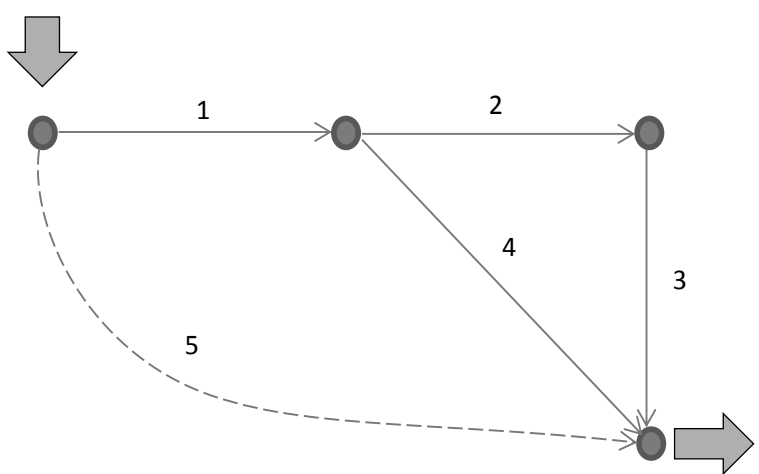

Figure 1. Test network 1 with an O-D pair

We assume the following mean travel time function, mean travel cost function, and variance and covariance of link travel time functions respectively given by

where

$$
\begin{gathered}
\vec{t}_{a}\left(E\left[V_{a}\right]\right)=0.1 \cdot \frac{E\left[V_{a}\right]}{E\left[C_{a}\right]}+\beta_{a} \quad \forall a \in \mathrm{A} \\
\vec{\rho}_{a}\left(E\left[V_{a}\right]\right)=0.5 \cdot \vec{t}_{a}\left(E\left[V_{a}\right]\right) \quad \forall a \in \mathrm{A} \\
\vec{\sigma}_{a}^{2}\left(E\left[V_{a}\right]\right)=\left(c v_{a} \cdot E\left[V_{a}\right]\right)^{2} \quad \forall a \in \mathrm{A} \\
\vec{\sigma}_{a b}\left(E\left[V_{a}\right], E\left[V_{b}\right]\right)=\breve{r}_{a b} \cdot \vec{\sigma}_{a}\left(E\left[V_{a}\right]\right) \cdot \vec{\sigma}_{b}\left(E\left[V_{b}\right]\right) \forall a, b \in \mathrm{A}
\end{gathered}
$$

$$
\begin{gathered}
E\left[C_{a}\right]=1 \\
c v_{a}=0.05 \\
\breve{r}_{a b}=0.5
\end{gathered}
$$

For simplicity, we set the variance of link travel time as equal to the variance of link flow. This setting will be adopted in the next numerical example.

The utility function of this problem is

$$
u\left(E\left[Q_{1}\right]\right)=\alpha_{1} \cdot \ln \left(E\left[Q_{1}\right]+1\right)
$$

where

$$
\alpha_{1}=1
$$

Parameters and observed link flows that are prepared considering neither predefined values of travel time and travel time reliability nor the equilibrium condition are shown in Table1.

Table 1. Observed link flows and assumed parameters

\begin{tabular}{|ccccc|}
\hline$a$ & 1 & 2 & 3 & 4 \\
\hline$E\left[\hat{V}_{a}\right]$ & 100 & 20 & 20 & 80 \\
\hline$\beta_{a}$ & 10 & 10 & 10 & 14 \\
\hline
\end{tabular}

Table 2. Estimates of route performance values

\begin{tabular}{|ccc|}
\hline route $(j)$ & $1(1->2->3)$ & $2(1->4)$ \\
\hline observed route flow $\left(E\left[\hat{F}_{1 j}\right]\right)$ & 20 & 80 \\
\hline mean travel time $\left(E\left[\breve{\Xi}_{1 j}\right]\right)$ & 44 & 42 \\
\hline mean travel cost $\left(E\left[\widetilde{\Gamma}_{1 j}\right]\right)$ & 22 & 21 \\
\hline travel time reliability $\left(\operatorname{var}\left[\breve{\Xi}_{1 j}\right]\right)$ & 38 & 61 \\
\hline
\end{tabular}


We assume $\breve{q}_{1}=200$, which results in $E\left[\hat{Q}_{1} \mid=100\right.$ and $e_{1}=100$. Table 2 shows the estimates of route performance values under the observed link flows. The constraints are estimated as $\phi=3380$, $\pi=1690$ and $\theta=49573$ from the observed link flows.

As explained earlier, an optimization problem that is equivalent to UE-ED cannot be formulated, due to the fact that covariance term $\vec{\sigma}_{a b}\left(E\left[V_{a}\right], E\left[V_{b}\right]\right) \neq 0$. Therefore, we solved a problem that is formulated by UE-NCP. It is well known that the solutions, i.e., route flows and the values of travel time and travel time reliability, are invariant to a positive monotonic transformation made to the utility function. In solving the problem, we applied the positive monotonic transformed direct utility function given by $\tilde{u}\left(E\left[Q_{1}\right]\right)=10^{5} \cdot u\left(E\left[Q_{1}\right]\right)$.

Table 3. Results of test network 1

\begin{tabular}{|ccccccc|c|}
\hline$\lambda^{*}$ & $\gamma^{*}$ & $\omega^{*}$ & $E\left[V_{1}^{*}\right]$ & $E\left[V_{2}^{*}\right]$ & $E\left[V_{3}^{*}\right]$ & $E\left[V_{4}^{*}\right]$ & $e_{1}^{*}$ \\
\hline 16.68 & 1.82 & 8.49 & 100 & 20 & 20 & 80 & 100 \\
\hline
\end{tabular}

The results are summarized in Table 3. Exactly the same link flows as the observed link flows were estimated in this experiment. The values of travel time and travel time reliability were estimated as $\tau=1.96$ and $v=0.21$. Even though the link flows that are equal to the observed ones are estimated, the parameters relating to the route performance value cannot be determined uniquely, since all sets of feasible parameters that satisfy the following conditions can generate equilibria.

$$
\begin{gathered}
\breve{c}_{11}(\mathbf{F})=\breve{c}_{12}(\mathbf{F})=d_{i}\left(e_{1}\right) \\
\Leftrightarrow \lambda \cdot E\left[\breve{\Xi}_{11}\right]+\omega \cdot E\left[\breve{\Gamma}_{11}\right]+\gamma \cdot \operatorname{var}\left[\breve{\Xi}_{11}\right]=\lambda \cdot E\left[\breve{\Xi}_{12}\right]+\omega \cdot E\left[\breve{\Gamma}_{12}\right]+\gamma \cdot \operatorname{var}\left[\breve{\Xi}_{12}\right]=d_{i}\left(e_{1}\right) \\
\Leftrightarrow 44 \cdot \lambda+22 \cdot \omega+38 \cdot \gamma=42 \cdot \lambda+21 \cdot \omega+61 \cdot \gamma=d_{i}\left(e_{1}\right)
\end{gathered}
$$

This fact is clear, since the number of unknown parameters is three, whereas the number of equations (or the number of equilibrated routes) is two.

\subsection{Experiment under Given Values of Travel Time and Travel Time Reliability}

This section addresses the link flows calculated based on both the predefined values of travel time and travel time reliability and the equilibrium condition. Also, the route performance value calculated by using the BPR function is employed. Figure 2 shows a test network with two O-D pairs in which the number of equilibrated routes is four, as shown by the sequence of link numbers, $1 \rightarrow 2 \rightarrow 5,1 \rightarrow 2 \rightarrow 6 \rightarrow$ $7,1 \rightarrow 3$ and $1 \rightarrow 4 \rightarrow 7$ between O-D pair 1 . There is an independent route, $8 \rightarrow 2$, between O-D pair 2 . The two dotted lines in Figure 2 are dummy links.

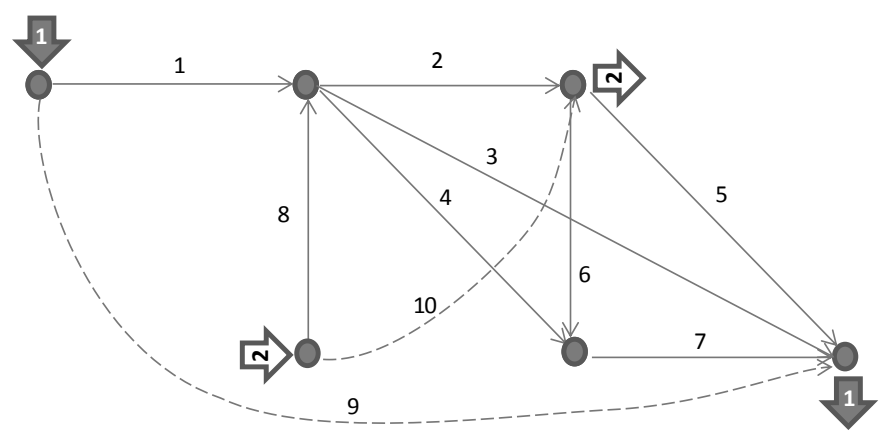

Figure 2. Test network 2 with two O-D pairs

We assume that the following mean travel time function, mean travel cost function, and variance and covariance of link travel time functions are respectively given by

$$
\vec{t}_{a}\left(E\left[V_{a}\right]\right)=\frac{l_{a}}{s_{0}} \cdot\left(1+m \cdot\left(\frac{E\left[V_{a}\right]}{E\left[C_{a}\right]}\right)^{n}\right) \forall a \in \mathrm{A}
$$




$$
\begin{gathered}
\vec{\rho}_{a}\left(E\left[V_{a}\right]\right)=p_{g} \cdot\left(r_{1} \cdot l_{a} \cdot\left(1+m \cdot\left(\frac{E\left[V_{a}\right]}{E\left[C_{a}\right]}\right)^{n}\right)+r_{2} \cdot l_{a}\right) \forall a \in \mathrm{A} \\
\vec{\sigma}_{a}^{2}\left(E\left[V_{a}\right]\right)=\left(c v_{a} \cdot E\left[V_{a}\right]\right)^{2} \quad \forall a \in \mathrm{A} \\
\vec{\sigma}_{a b}\left(E\left[V_{a}\right], E\left[V_{b}\right]\right)=\breve{r}_{a b} \cdot \vec{\sigma}_{a}\left(E\left[V_{a}\right]\right) \cdot \vec{\sigma}_{b}\left(E\left[V_{b}\right]\right) \forall a, b \in \mathrm{A}
\end{gathered}
$$

where $l_{a}$ is length of link $a ; s_{0}$ is free flow speed $(60[\mathrm{~km} / \mathrm{hr}]) ; m$ and $n$ are parameters for the BPR function $(m=5, n=2) ; p_{g}$ is gas price (140[JPY/l]); $r_{1}=0.009$ and $r_{2}=0.059$ are the parameters for travel cost function estimated in Uchida (2010); $c v_{a}=0.5$; and we prepare two cases

$$
\breve{r}_{a b}=\left\{\begin{array}{cc}
0 & \text { for case } 1 \text { (independent) } \\
0.1 & \text { for case } 2 \text { (correlatel) }
\end{array}\right.
$$

A relatively larger parameter $m$ than that estimated in the real traffic situation is set, to see easily how the equilibrium variables change with change in observed flow. The utility function of this problem is

$$
u\left(E\left[Q_{1}\right], E\left[Q_{2}\right]\right)=\alpha_{1} \cdot \ln \left(E\left[Q_{1}\right]+1\right)+\alpha_{2} \cdot \ln \left(E\left[Q_{2}\right]+1\right)
$$

where $\left(\alpha_{1}, \alpha_{2}\right)$ are calculated from (B.9) in Appendix B as $(0.96,0.04)$ in case 1 and as $(0.97,0.03)$ in case 2 . We used $\tilde{u}\left(E\left[Q_{1}\right], E\left[Q_{2}\right]\right)$ which is obtained by applying a transformation similar to that applied to $u\left(E\left[Q_{1}\right]\right)$ to $u\left(E\left[Q_{1}\right], E\left[Q_{2}\right]\right)$.

The equilibrated link flows that are assumed as the observed link flows are calculated based on the value of time, 40 [JPY/min], and the value of travel time reliability, 20 [JPY/unit variance], as shown in Table 4. The budget constraints, $\phi, \pi$ and $\theta$, are estimated by using the observed link flows. We assume $\breve{q}_{1}=400$ and $\breve{q}_{2}=100$, which results in $E\left[\hat{Q}_{1}\right]=320$ and $e_{1}=80$, and in $E\left[\hat{Q}_{2}\right\rfloor=50$ and $e_{2}=50$. Table 5 and Table 6 show the estimates of route performance values under the coefficients of correlation of $\breve{r}_{a b}=0$ and $\breve{r}_{a b}=0.1$, respectively. It is shown that the two sets of observed link flows under the two cases satisfy the equilibrium condition.

Table 4. Observed link flows and assumed parameters

\begin{tabular}{|crrrrrrrr|}
\hline$a$ & 1 & 2 & 3 & 4 & 5 & 6 & 7 & 8 \\
\hline$E\left[\hat{V}_{a}\right]\left(\breve{r}_{a b}=0\right)$ & 320.0 & 160.2 & 110.1 & 99.8 & 70.8 & 39.4 & 139.1 & 50.0 \\
\hline$E\left[\hat{V}_{a}\right]\left(\breve{r}_{a b}=0.1\right)$ & 320.0 & 145.8 & 122.0 & 102.1 & 75.9 & 19.9 & 122.1 & 50.0 \\
\hline$E\left[C_{a}\right]$ & 1500 & 2000 & 500 & 700 & 1500 & 1500 & 1500 & 1500 \\
\hline$l_{a}[\mathrm{~km}]$ & 10 & 10 & 50 & 30 & 30 & 5 & 10 & 10 \\
\hline
\end{tabular}

Table 5. Estimates of route performance values (case 1: $\breve{r}_{a b}=0$ )

\begin{tabular}{|rrrrrr|}
\hline $\begin{array}{r}\text { route } i, j \\
\text { (link seq. })\end{array}$ & $\begin{array}{c}1,1 \\
(1 \rightarrow 2 \rightarrow 5)\end{array}$ & $\begin{array}{c}1,2 \\
(1 \rightarrow 2 \rightarrow 6 \rightarrow 7)\end{array}$ & $\begin{array}{c}1,3 \\
(1 \rightarrow 3)\end{array}$ & $\begin{array}{c}1,4 \\
(1 \rightarrow 4 \rightarrow 7)\end{array}$ & \multicolumn{1}{c|}{\begin{tabular}{l}
$(8 \rightarrow 2)$ \\
\hline$E\left[\hat{F}_{i j}\right]$
\end{tabular}} \\
\hline$E\left[\breve{\Xi}_{i j}\right]$ & 70.8 & 39.4 & 110.1 & 99.8 & 50.0 \\
\hline$E\left[\breve{\Gamma}_{i j}\right]$ & 54.1 & 38.0 & 74.4 & 55.8 & 20.4 \\
\hline $\operatorname{var}\left[\breve{\Xi}_{i j}\right]$ & 506.8 & 355.0 & 623.6 & 509.5 & 200.6 \\
\hline$\breve{c}_{i j}(\mathbf{F})\left[\times 10^{2}\right]^{\#}$ & 332.7 & 372.4 & 286.4 & 329.3 & 70.4 \\
\hline & $\mathbf{9 3 . 3}$ & $\mathbf{9 3 . 3}$ & $\mathbf{9 3 . 3}$ & $\mathbf{9 3 . 3}$ & 24.2 \\
\hline
\end{tabular}


Table 6. Estimates of route performance values (case 2: $\breve{r}_{a b}=0.1$ )

\begin{tabular}{|rrrrrr|}
\hline $\begin{array}{c}\text { route } i, j \\
\text { (link seq. })\end{array}$ & $\begin{array}{c}1,1 \\
(1 \rightarrow 2 \rightarrow 5)\end{array}$ & $\begin{array}{c}1,2 \\
(1 \rightarrow 2 \rightarrow 6 \rightarrow 7)\end{array}$ & $\begin{array}{c}1,3 \\
(1 \rightarrow 3)\end{array}$ & $\begin{array}{c}1,4 \\
(1 \rightarrow 4 \rightarrow 7)\end{array}$ & \begin{tabular}{c} 
(8 $\rightarrow 2)$ \\
\hline$E\left[\hat{F}_{i j}\right]$
\end{tabular} \\
\hline$E\left[\breve{\Xi}_{i j}\right]$ & 78.7 & 24.2 & 116.5 & 100.6 & 50.0 \\
\hline$E\left[\breve{\Gamma}_{i j}\right]$ & 54.3 & 37.9 & 77.2 & 55.8 & 20.3 \\
\hline $\operatorname{var}\left[\breve{\Xi}_{i j}\right]$ & 507.0 & 354.7 & 628.1 & 509.5 & 200.5 \\
\hline$\breve{c}_{i j}(\mathbf{F})\left[\times 10^{2}\right]^{\#}$ & 364.6 & 405.0 & 312.8 & 361.4 & 63.1 \\
\hline & $\mathbf{9 9 . 7}$ & $\mathbf{9 9 . 7}$ & $\mathbf{9 9 . 7}$ & $\mathbf{9 9 . 7}$ & 22.7 \\
\hline
\end{tabular}

We will see how the equilibrium variables change with change in the observed flow. Figure 3 and Figure 4 show the estimated value of travel time and of travel time reliability (VOT and VOR, respectively) in case 1 and case 2, respectively, when the observed route flow, $E\left[\hat{F}_{21}\right]$, varies around $E\left[\hat{F}_{21}\right]=50$ where the same $\alpha_{1}$ and $\alpha_{2}$ as estimated by using the observed link flows in Table 4 are applied. It is shown that the model presented in this study estimates correctly the predefined value of travel time, 40 [JPY/min], and the predefined value of travel time reliability, 20 [JPY/unit variance], from two sets of observed traffic flows at $E\left[\hat{F}_{21}\right]=50$. Also, it is shown that the value of travel time and of travel time reliability increase with increase in the congestion level of the network (or $E\left[\hat{F}_{21}\right]$ ) in both cases. In addition, it is shown that the rate of increase for the values of travel time and travel time reliability are higher in case 1 than in case 2.

Table 7 and Table 8 show the equilibrium route performance values and the estimated parameters relating to the value of travel time and of travel time reliability in case 1 and case 2, respectively. Interestingly, it is shown that the equilibrium route performance value at each O-D pair does not change much when $E\left[\hat{F}_{21}\right]$ changes in either cases. However, the parameters relating to both values change as $E\left[\hat{F}_{21}\right]$ changes. In case $1, \lambda^{*}$ increases with increase in $E\left[\hat{F}_{21}\right]$; however, $\omega^{*}$ decreases with increase in $E\left[\hat{F}_{21}\right] \cdot \gamma^{*}$ is invariant to the change in $E\left[\hat{F}_{21}\right]$. In case $2, \lambda^{*}$ increases with increase in $E\left[\hat{F}_{21}\right]$; however, both $\omega^{*}$ and $\gamma^{*}$ decrease with increase in $E\left[\hat{F}_{21}\right]$. The rates of change for $\lambda^{*}$ and $\omega^{*}$ are higher in case 1 than in case 2 . In addition, $\gamma^{*}$ decreases with increase in $E\left[\hat{F}_{21}\right\rfloor$ in case 2, whereas $\gamma^{*}$ does not change in case 1 . As a result, the rates of increase of the value of travel time and of travel time reliability are higher in case 1 than in case 2.

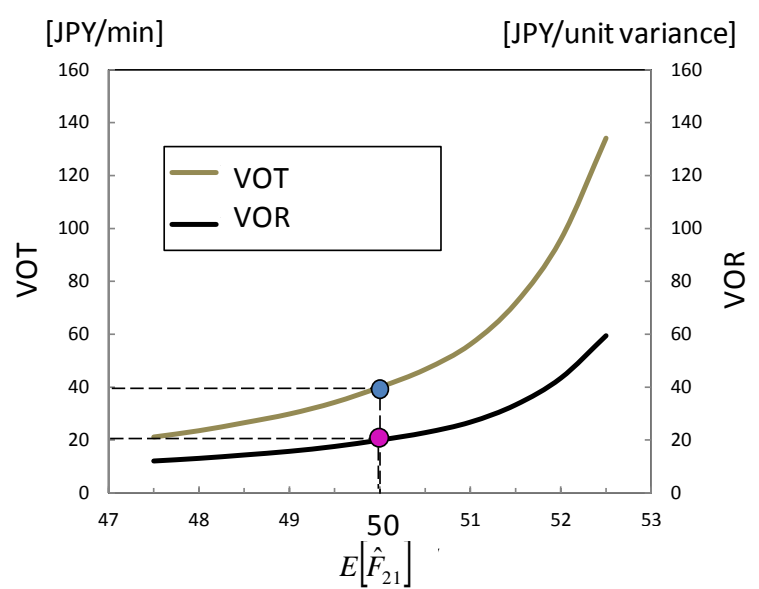

Figure 3. Estimated VOT and VOR (case1) 


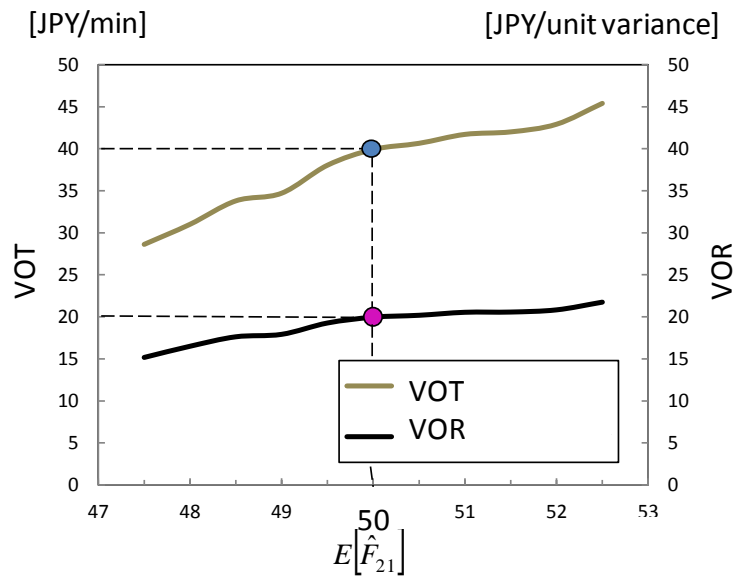

Figure 4. Estimated VOT and VOR (case2)

Table 7. Estimated variables (case 1)

\begin{tabular}{|crrrrrrrrrrr|}
\hline$E\left[\hat{F}_{21}\right]$ & 47.5 & 48.0 & 48.5 & 49.0 & 49.5 & 50.0 & 50.5 & 51.0 & 51.5 & 52.0 & 52.5 \\
\hline$d_{1}\left(e_{1}^{*}\right)\left[\times 10^{2}\right]$ & 93.4 & 93.4 & 93.4 & 93.3 & 93.3 & 93.3 & 93.2 & 93.2 & 93.2 & 93.1 & 93.1 \\
\hline$d_{2}\left(e_{2}^{*}\right)\left[\times 10^{2}\right]$ & 24.4 & 24.4 & 24.3 & 24.3 & 24.3 & 24.2 & 24.2 & 24.2 & 24.1 & 24.2 & 24.0 \\
\hline \hline$\lambda^{*}$ & 35.0 & 35.9 & 37.0 & 37.9 & 38.9 & 40.0 & 41.0 & 42.0 & 43.1 & 44.2 & 45.2 \\
\hline$\omega^{*}$ & 1.7 & 1.5 & 1.4 & 1.3 & 1.1 & 1.0 & 0.9 & 0.7 & 0.6 & 0.5 & 0.3 \\
\hline$\gamma^{*}$ & 20.0 & 20.0 & 20.0 & 20.0 & 20.0 & 20.0 & 20.0 & 20.0 & 20.0 & 20.0 & 20.0 \\
\hline
\end{tabular}

Table 8. Estimated variables (case 2)

\begin{tabular}{|crrrrrrrrrrr|}
\hline$E\left[\hat{F}_{21}\right]$ & 47.5 & 48.0 & 48.5 & 49.0 & 49.5 & 50.0 & 50.5 & 51.0 & 51.5 & 52.0 & 52.5 \\
\hline$d_{1}\left(e_{1}^{*}\right)\left[\times 10^{2}\right]$ & 100.0 & 99.9 & 99.9 & 99.8 & 99.7 & 99.7 & 99.5 & 99.7 & 99.9 & 99.5 & 99.1 \\
\hline$d_{2}\left(e_{2}^{*}\right)\left[\times 10^{2}\right]$ & 22.7 & 22.7 & 22.7 & 22.8 & 22.7 & 22.7 & 22.7 & 22.7 & 22.9 & 22.7 & 22.7 \\
\hline \hline$\lambda^{*}$ & 37.8 & 37.9 & 38.6 & 38.9 & 39.6 & 40.0 & 40.1 & 40.4 & 40.7 & 40.7 & 41.0 \\
\hline$\omega^{*}$ & 1.2 & 1.2 & 1.1 & 1.1 & 1.0 & 1.0 & 1.0 & 1.0 & 1.0 & 1.0 & 0.9 \\
\hline$\gamma^{*}$ & 20.3 & 20.2 & 20.2 & 20.1 & 20.0 & 20.0 & 19.9 & 19.9 & 19.9 & 19.7 & 19.6 \\
\hline
\end{tabular}

\section{CONCLUDING REMARKS}

This study proposed two network models that estimated the value of travel time and of travel time reliability in road networks. The proposed models assume that traffic flows in the network can be observed (or estimated). The estimated value of travel time and of travel time reliability are consistent with the observed traffic flows by taking account of the risk-averse driver's route choice behaviour. The proposed models have the same structures as the UE traffic assignment problem with elastic demand. Under the separable and monotonically increasing link performance function, the value of travel time and of travel time reliability can be estimated by solving an optimization problem that is equivalent to a standard UE traffic assignment problem. In general, the risk-averse driver's route choice behaviour cannot be formulated as an optimization problem, since the link performance function is nether separable nor monotonically increasing. Therefore, we formulated a nonlinear complementary problem under such situation for estimating the value of travel time and of travel time reliability. The O-D demand functions formulated in the proposed models were derived from the utility maximization behaviour of the driver in the network. Therefore, the O-D demand function is consistent with the studies that address the value of travel time and of travel time reliability based on utility maximization behaviour without considering the driver's route choice. 
Two numerical experiments under the non-separable link performance function were carried out to demonstrate the proposed models. The number of O-D pairs was one in the first experiment and two in the second experiment. It is shown that the models proposed in this study can correctly estimate the values of travel time and travel time reliability. As expected, it was clarified that the value of travel time and of travel time reliability in the network both increased with increase in congestion level. This fact is supported by the literature but has not been demonstrated in a network-level problem. It should be noted that this fact was obtained from experiments that explicitly addressed the risk-averse driver's route choice behaviour.

Since the value of travel time and of travel time reliability can be different for each O-D pair, the formulation of a model under the O-D specific value of travel time and of travel time reliability may be a remaining research topic. An extension of the proposed models to a dynamic framework, i.e., the time-varying values of travel time and travel time reliability, is an interesting topic. The first research topic can be carried out. In the near future, we will present it. The second research topic is challenging but needs to be addressed in the research area of the time-varying congestion charging problem.

We provided two strong assumptions in formulating the models, i.e., $V_{a}$ (or $C_{a}$ ) follows a theoretical distribution that is characterized only by $E\left[V_{a}\right]$ and $\operatorname{var}\left[V_{a}\right]$ (or, $E\left[C_{a}\right]$ and $\operatorname{var}\left[C_{a}\right]$ ), and the Taylor series expansion can approximate the link travel time and the variance of two link travel times with satisfactory accuracy. These two assumptions allow us to provide the simplified formulation of the models. However, these assumptions need to be relaxed for the development of a plausible model, which is our future task.

\section{Appendix A}

By performing the $m$ th-order Taylor series expansion to $t_{a}\left(V_{a} ; C_{a}\right)$ at $V_{a}=E\left[V_{a}\right]$ and $C_{a}=E\left[C_{a}\right]$, we obtain

$$
t_{a}\left(V_{a} ; C_{a}\right) \approx \sum_{0 \leq i+j \leq m} b_{i j a} \cdot\left(V_{a}-E\left[V_{a}\right]\right)^{i} \cdot\left(C_{a}-E\left[C_{a}\right]\right)^{j}
$$

where

$$
b_{i j a}=\left.\frac{1}{i ! \cdot j !} \cdot \frac{\partial^{i+j} t_{a}\left(V_{a} ; C_{a}\right)}{\partial\left(V_{a}\right)^{i} \partial\left(C_{a}\right)^{j}}\right|_{V_{a}=E\left[V_{a}\right], C_{a}=E\left[C_{a}\right]}
$$

Since $V_{a}$ and $C_{a}$ are independently distributed, we obtain

$$
E\left[t_{a}\left(V_{a} ; C_{a}\right)\right] \approx \sum_{0 \leq i+j \leq m} b_{i j a} \cdot c_{j a} \cdot E\left[\left(V_{a}-E\left[V_{a}\right]\right)^{i}\right]
$$

where

$$
c_{j a}=E\left[\left(C_{a}-E\left[C_{a}\right]\right)^{j}\right]
$$

Note that in a traffic assignment problem, $c_{j a}$ can be regarded as constant terms that are expressed by the functions of $E\left[C_{a}\right]$ and $\operatorname{var}\left[C_{a}\right]$ based on the assumption in section 2.2. In a similar way, the $i$ thorder moment of $V_{a}, E\left[\left(V_{a}-E\left[V_{a}\right]\right)^{i}\right]$, is expressed by a function of $E\left[V_{a}\right]$ and $\operatorname{var}\left[V_{a}\right]=\left(c v_{a} \cdot E\left[V_{a}\right]\right)^{2}$. Thus, we obtain

$$
E\left[t_{a}\left(V_{a} ; C_{a}\right)\right] \approx \vec{t}_{a}\left(E\left[V_{a}\right]\right)
$$

For the calculation of the covariance of two link travel times, we consider first $t_{a}\left(V_{a} ; C_{a}\right) \cdot t_{b}\left(V_{b} ; C_{b}\right)$, which can be given by

$$
\sum_{0 \leq i_{1}+j_{1} \leq m}^{t_{a}\left(V_{a} ; C_{a}\right) \cdot t_{b}\left(V_{b} ; C_{i_{2}}+j_{2} \leq m\right.} b_{i_{1} j_{1}} \cdot b_{i_{2} j_{2} b} \cdot\left(V_{a}-E\left[V_{a}\right]\right)^{i_{1}} \cdot\left(V_{b}-E\left[V_{b}\right]\right)^{i_{2}} \cdot\left(C_{a}-E\left[C_{a}\right]\right)^{j_{1}} \cdot\left(C_{b}-E\left[C_{b}\right]\right)^{j_{2}}
$$

By applying the same discussion above, we obtain 
where

$$
E\left[t_{a}\left(V_{a} ; C_{a}\right) \cdot t_{b}\left(V_{b} ; C_{b}\right)\right] \approx \sum_{0 \leq i_{1}+j_{1} \leq m} \sum_{0 \leq i_{2}+j_{2} \leq m} b_{i_{1} j_{1} a} \cdot b_{i_{2} j_{2} b} \cdot c_{j_{1} j_{2} a b} \cdot E\left[\left(V_{a}-E\left[V_{a}\right]\right)^{i_{1}} \cdot\left(V_{b}-E\left[V_{b}\right]\right)^{i_{2}}\right]
$$

$$
c_{j_{1} j_{2} a b}=E\left[\left(C_{a}-E\left[C_{a}\right]\right)^{j_{1}} \cdot\left(C_{b}-E\left[C_{b}\right]\right)^{j_{2}}\right]
$$

Note that in a traffic assignment problem, $c_{j_{1} j_{2} a b}$ can be regarded as constant terms that are expressed by the functions of $E\left[C_{a}\right], E\left[C_{b}\right]$ and $\operatorname{cov}\left[C_{a}, C_{b}\right]$ based on the assumption in section 2.2. In a similar way, $E\left[\left(V_{a}-E\left[V_{a}\right]\right)^{i_{1}} \cdot\left(V_{b}-E\left[V_{b}\right]\right)^{i_{2}}\right]$ are expressed by the functions of $E\left[V_{a}\right], E\left[V_{b}\right]$ and $\operatorname{cov}\left[V_{a}, V_{b}\right]=r_{a b} \cdot c v_{a} \cdot c v_{b} \cdot E\left[V_{a}\right] \cdot E\left[V_{b}\right]$. Thus, we obtain

$$
\sigma_{a b}\left(V_{a}, V_{b} ; C_{a}, C_{b}\right) \approx E\left[t_{a}\left(V_{a} ; C_{a}\right) \cdot t_{b}\left(V_{b} ; C_{b}\right)\right]-\vec{t}_{a}\left(E\left[V_{a}\right]\right) \cdot \vec{t}_{a}\left(E\left[V_{b}\right]\right)=\vec{\sigma}_{a b}\left(E\left[V_{a}\right], E\left[V_{b}\right]\right)
$$

\section{Appendix B}

The utility maximization problem of driver $k$ is given by

$$
\begin{gathered}
\max u_{k}\left(q_{1}^{k}, \ldots, q_{|I|}^{k}\right)=\sum_{i \in \mathrm{I}} \int_{0}^{q_{i}^{k}} \frac{\alpha_{i}^{k}}{w+1} \cdot d w \\
\sum_{i \in \mathrm{I}} t_{i} \cdot q_{i}^{k} \leq \phi_{k} \\
\sum_{i \in \mathrm{I}} \rho_{i} \cdot q_{i}^{k} \leq \pi_{k} \\
\sum_{i \in \mathrm{I}} \sigma_{i}^{2} \cdot q_{i}^{k} \leq \theta_{k}
\end{gathered}
$$

A variable with the superscript (or the subscript) $k$ means that the variable is specific to driver $k$. The budget constraints shown by (B.2)-(B.4) can be written as

where

$$
\sum_{i \in \mathrm{I}} g_{i} \cdot q_{i}^{k} \leq \phi_{k}+\pi_{k}+\theta_{k}
$$

$$
g_{i}=t_{i}+\rho_{i}+\sigma_{i}^{2}
$$

The Lagrangian function for the utility maximization problem is

$$
\max l_{k}\left(q_{1}^{k}, \ldots, q_{|| \mid}^{k}, \mu_{k}\right)=\sum_{i \in \mathrm{I}} \alpha_{i}^{k} \cdot \ln \left(q_{i}^{k}+1\right)+\mu_{k} \cdot\left(\phi_{k}+\pi_{k}+\theta_{k}-\sum_{i \in \mathrm{I}} g_{i} \cdot q_{i}^{k}\right)
$$

where $\mu_{k}$ is the Lagrangian multiplier. The first-order conditions for the Lagrangian function are

$$
\begin{gathered}
\frac{\partial l_{k}\left(q_{1}^{k}, \ldots, q_{|| \mid}^{k}, \mu_{k}\right)}{\partial q_{i}^{k}}=\frac{\alpha_{i}^{k}}{q_{i}^{k}+1}-\mu_{k} \cdot g_{i}=0 \\
\frac{\partial l_{k}\left(q_{1}^{k}, \ldots, q_{|| \mid}^{k}, \mu_{k}\right)}{\partial \mu_{k}}=\phi_{k}+\pi_{k}+\theta_{k}-\sum_{i \in \mathrm{I}} g_{i} \cdot q_{i}^{k}=0
\end{gathered}
$$

By solving (B.5) and (B.6), we obtain

$$
q_{i}^{k^{*}}=\frac{\alpha_{i}^{k} \cdot\left(\phi_{k}+\pi_{k}+\theta_{k}\right)}{g_{i}}
$$

Since $\alpha_{i}^{k}$ is the distribution parameter for the Cobb-Douglas utility function, the parameter is given by (Varian, 1984)

Therefore, we obtain

$$
\alpha_{i}^{k}=\frac{g_{i} \cdot \hat{q}_{i}^{k}}{\phi_{k}+\pi_{k}+\theta_{k}}
$$

$$
q_{i}^{k^{*}}=\hat{q}_{i}^{k}
$$


The observed or estimated O-D flows are then

$$
\hat{q}_{i}=\sum_{k} \hat{q}_{i}^{k}=\sum_{k} \frac{\alpha_{i}^{k} \cdot\left(\phi_{k}+\pi_{k}+\theta_{k}\right)}{g_{i}}=\frac{\phi+\pi+\theta}{g_{i}} \cdot \sum_{k} \alpha_{i}^{k} \cdot \frac{\phi_{k}+\pi_{k}+\theta_{k}}{\phi+\pi+\theta}=\frac{\alpha_{i} \cdot(\phi+\pi+\theta)}{g_{i}}
$$

where

$$
\alpha_{i}=\sum_{k} \alpha_{i}^{k} \cdot \frac{\phi_{k}+\pi_{k}+\theta_{k}}{\phi+\pi+\theta}
$$

By substituting (B.6) into (B.7), we obtain

$$
\alpha_{i}=\frac{g_{i} \cdot \hat{q}_{i}}{\phi+\pi+\theta}
$$

By comparing (B.7) and (B.9), and recalling that the parameter shown by (B.7) corresponds to the utility function shown by (B.1), it is clear that $\alpha_{i}$ in (B.9) represents the parameter corresponding to the utility function (17). Thus, by aggregating the drivers' utility maximization behaviours, we obtain the model presented in section 4.2.

\section{Appendix C}

The CES utility function corresponding to (17) is given by

$$
u\left(q_{1}, \ldots, q_{|I|}\right)=\left(\sum_{i \in \mathrm{I}}\left(\alpha_{i}\right)^{\frac{1}{\varepsilon_{1}}} \cdot\left(q_{i}+1\right)^{1-\frac{1}{\varepsilon_{1}}}\right)^{\frac{1}{\varepsilon_{1}-1}}
$$

$\alpha_{i}$ are the distribution parameters satisfying

$$
\sum_{i \in \mathrm{I}}\left(\alpha_{i}\right)^{\frac{1}{\varepsilon_{1}}}=1
$$

where $\varepsilon_{1}$ is substitution elasticity. If $\varepsilon_{1}=1$, then we obtain the same Cobb-Douglas utility function (Varian, 1984) as (17) given by

$$
u\left(q_{1}, \ldots, q_{|I|}\right)=\sum_{i \in \mathrm{I}} \alpha_{i} \cdot \ln \left(q_{i}+1\right)
$$

where

$$
\sum_{i \in \mathrm{I}} \alpha_{i}=1
$$

The expense share for goods $q_{i}$ is fixed in the Cobb-Douglas utility function.

We introduce the composite goods, $z$, which represents all goods other than those relating to the road transportation market. We select $z$ as a numeraire with the price of one. We apply the maximization problem of quasilinear utility function for the nested CES utility function given by

$$
\max u(z, q)=z+\alpha_{q} \cdot(q)^{\frac{\varepsilon_{0}-1}{\varepsilon_{0}}}
$$

s.t.

$$
z+\sum_{i \in \mathrm{I}} g_{i} \cdot q_{i} \leq \eta
$$

where

$$
q=\left(\sum_{i \in \mathrm{I}}\left(\alpha_{i}\right) \frac{1}{\varepsilon_{1}} \cdot\left(q_{i}+1\right)^{1-\frac{1}{\varepsilon_{1}}}\right)^{\frac{1}{\varepsilon_{1}-1}}
$$

$\varepsilon_{0}$ is substitution elasticity and $\eta$ is a budget. $\alpha_{q}$ is a parameter to be formulated. The nested CES utility maximization problem can be solved in two stages. In the second stage, we solve the following problem 


$$
\max q\left(q_{1}, \ldots, q_{|I|}\right)=\left(\sum_{i \in \mathrm{I}}\left(\alpha_{i}\right)^{\frac{1}{\varepsilon_{1}}} \cdot\left(q_{i}+1\right)^{1-\frac{1}{\varepsilon_{1}}}\right)^{\frac{1}{\varepsilon_{1}-1}}
$$

s.t.

Then we obtain the demand function

$$
\sum_{i \in \mathrm{I}} g_{i} \cdot q_{i} \leq \phi+\pi+\theta
$$

$$
q_{i}=\frac{\alpha_{i}}{\left(g_{i}\right)^{\varepsilon_{1}}} \cdot \frac{\phi+\pi+\theta}{\sum_{j \in \mathrm{I}} \alpha_{j} \cdot\left(g_{j}\right)^{1-\varepsilon_{1}}}
$$

Next we find the price for goods relating to the transportation market, $g$, such that $g=(\phi+\pi+\theta) / q$. From (C.1) and (C.2), we obtain

$$
q=\left(\sum_{i \in \mathrm{I}} \alpha_{i} \cdot\left(g_{i}\right)^{1-\varepsilon_{1}}\right)^{\frac{1}{\varepsilon_{1}-1}} \cdot(\phi+\pi+\theta)
$$

Therefore

$$
g=\left(\sum_{i \in \mathrm{I}} \alpha_{i} \cdot\left(g_{i}\right)^{1-\varepsilon_{1}}\right)^{\frac{1}{\varepsilon_{1}-1}}
$$

In the first stage, by using $g$ shown by (C.3), the following utility maximization problem is solved

$$
\max u(z, q)=z+\alpha_{q} \cdot(q)^{\frac{\varepsilon_{0}-1}{\varepsilon_{0}}}
$$

s.t.

$$
z+g \cdot q \leq \eta
$$

By solving the above utility maximization problem, we obtain the following demand functions

$$
\begin{gathered}
z=\eta-\left(\frac{\varepsilon_{0}-1}{\varepsilon_{0}} \cdot \frac{\alpha_{q}}{g}\right)^{\varepsilon_{0}} \cdot g \\
q=\left(\frac{\varepsilon_{0}-1}{\varepsilon_{0}} \cdot \frac{\alpha_{q}}{g}\right)^{\varepsilon_{0}}
\end{gathered}
$$

Note that, $q$ is independent of $\eta$. An effect of the transportation market, e.g., a change in the price of goods relating to the transportation market, $g$, on all other markets which are represented by the composite goods can be estimated from above two demand functions.

If $\varepsilon_{1}=1$ in the second-stage problem, the models proposed in this study are obtained as follows.

$$
q_{i}=\frac{\alpha_{i} \cdot(\phi+\pi+\theta)}{g_{i}}
$$

The remaining parameter, $\alpha_{q}$, is given by (Fujiwara et al., 2004)

$$
\alpha_{q}=\frac{\varepsilon_{0}-1}{\varepsilon_{0}} \cdot q^{\frac{1}{\varepsilon_{0}}} \cdot g
$$

\section{REFERENCES}

Asakura, Y., Kashiwadani, M. 1991. Road network reliability caused by daily fluctuation of traffic flow, Proceedings of the $19^{\text {th }}$ PTRC Summer Annual Meeting in Brighton, Seminar G, 73-84. Becker, G.S., 1965. A theory of the allocation of time. The Economic Journal 75 (299), 493-517. 
Bell, M.G.H., Cassir, C., Iida, Y., Lam, W.H.K., 1993. A sensitivity-based approach to network reliability assessment, Proceedings of the 14th International Symposium on Transportation and Traffic Theory, Jerusalem, Israel, 283-300.

Börjesson, B., Eliasson, J., Franklin, J.P., 2012. Valuations of travel time variability in scheduling versus mean-variance models, Transportation Research Part B 46, 855-873.

Bras, R.L., Georgakakos, K.P., 1989. Real-time nonlinear filtering techniques in streamflow forecasting: a statistical linearization approach. Proceedings of the Third International Symposium on Stochastic Hydraulics, 95-105.

Brownstone, D., Small, K.A., 2005. Valuing Time and Reliability: Assessing the evidence from road pricing demonstrations, Transportation Research Part A 39, 279-293.

Cascetta, E., 1989. A stochastic process approach to the analysis of temporal dynamics in transportation networks. Transportation Research 23B(1), 1-17.

Cascetta, E., Canterella, G. E., 1991. A day-to-day and within-day dynamic stochastic assignment model. Transportation Research 25A, 277-291.

Cassir, C., Bell, M.G.H., 2002. Estimation of Travel Time Reliability Using Stochastic User Equilibrium Assignment Sensitivity, Transportation Planning. eds M. Patriksson and M. Labbé, Kluwer Academic Publishers, UK, 69-84.

Chen, A., Yang, H., Lo, H.K., Tang, W.H., 1999. A capacity related reliability for transportation networks. Journal of Advanced Transportation, 33 (2), 183-200.

Chen, A., Yang, H., Lo, H.K., Tang, W.H., 2002. Capacity reliability of a road network: an assessment methodology and numerical results. Transportation Research, 36B (3), 225-252.

Chen, B.Y., Lam, W.H.K., Sumalee, A., Shao, H., 2011. An efficient solution algorithm for solving multi-class reliability-based traffic assignment problem. Mathematical and Computer Modelling, 54, 1428-1439.

Chen, A., Zhou, Z., 2010. The $\alpha$-reliable mean-excess traffic equilibrium model with stochastic travel times. Transportation Research Part B 44 (4), 493-513.

Chen, A., Zhou, Z., Lam, W.H.K., 2011. Modeling stochastic perception error in the mean-excess traffic equilibrium model. Transportation Research Part B 45, 1619-1640.

Clark, S.D., Watling, D.P., 2005. Modelling network travel time reliability under stochastic demand. Transportation Research, 39B (2), 119-140.

Cobb, C.W., Douglas, P.H., 1928. A theory of production. American Economic Review, 18 (Supplement), 139-165.

DeSerpa, A.C., 1971. A theory of the economics of time. The Economic Journal, 81(324), 828-846.

Fosgerau, M., Karlström, M., 2010. The value of reliability. Transportation Research Part B 44, 38-49.

Fosgerau, M., Engelson, L., 2011. The value of travel time variance. Transportation Research Part B (45), 1-8.

Fujiwara, T., Hasuike, K., Kanemoto Y., 2004. A small-scale microeconomic model for policy evaluation - evaluating measures to combat global warming in the passenger vehicle sector. RIETI Discussion Paper Series 04-J-046 (in Japanese).

Gartner, G.H., 1980. Optimal traffic assignment with elastic demands: A review part II, Transportation Science, 14, pp. 174-191.

Hensher, D.A., Greene, W.H., Li, Z., 2011. Embedding risk attitude and decision weights in non-linear logit to accommodate time variability in the value of expected travel time savings. Transportation Research Part B 45, 954-972.

Isserlis, L., 1918. On a formula for the product-moment coefficient of any order of normal frequency distribution in any number of variables. Biometrika, 12 (1-2), 134-139.

Lam, W.H.K., Shao, H., Sumalee, A., 2008. Modeling impacts of adverse weather conditions on a road network with uncertainty in demand and supply. Transportation Research Part B, 42(10), 890-910.

Lam, T., Small, K., 2001. The value of time and reliability: measurement from a value pricing experiment. Transportation Research Part E 37, 231-251.

Lasdon, L., 1970. Optimization theory for large systems. Macmillian, New York.

Lo, H.K., Chen, A., 2000. Traffic equilibrium problem with route-specific costs: Formulation and algorithms. Transportation Research, 34B(6), 493-514.

Lo, H.K., Tung, Y.K., 2003. Network with degradable links: capacity analysis and design. Transportation Research, 37B (4), 345-363. 
Nakayama, S., Takayama, J., 2003. Traffic network equilibrium model for uncertain demands. The 82nd Annual Meeting of the Transportation Research Board, Washington, D.C.

Ng, M.W., Szeto, W.Y., Waller, S.T., 2011. Distribution-free travel time reliability assessment with probability inequalities. Transportation Research Part B, 45, 852-866.

Ng, M.W., Waller, S.T., 2010. A computationally efficient methodology to characterize travel time reliability using the fast Fourier transform. Transportation Research Part B 44, 1202-1219.

Shao, H., Lam, W.H.K., Tam, M.L., 2006. A reliability-based stochastic traffic assignment model for network with multiple user classes under uncertainty in demand. Networks and Spatial Economics, 6(3-4), 173-204.

Shao, H., Lam, W.H.K., Tam, M., Yuan, X.M., 2008. Modelling rain effects on risk-taking behaviours of multi-user classes in road networks with uncertainty. Journal of Advanced Transportation, 42(3), 265-290.

Sherali, H.D., Narayanan, A., Sivanandan, R., 2003. Estimation of origin-destination trip-tables based on a partial set of traffic link volumes. Transportation Research Part B 37, 815-836.

Sumalee, A., Uchida, K., Lam, W.H.K., 2011. Stochastic multi-modal transport network under demand uncertainties and adverse weather condition. Transportation Research Part C 19, 338-350.

Sumalee, A., Xu, W., 2011. First-best marginal cost toll for a traffic network with stochastic demand, Transportation Research Part B, 45(1), 41-59.

Uchida, K., 2010. Equilibrium traffic assignment models based on the $\mathrm{CO} 2$ emission minimization principle. Selected papers on Environmental Systems Japan Society of Civil Engineers 38, 101108 (in Japanese).

Uchida, K., Munehiro, K., 2010. Impact of stochastic traffic capacity on travel time in road network. The 89th Annual Meeting of the Transportation Research Board, Washington, D.C.

Van der Zijpp, N.J., Fiorenzo Catalano, M.S., 2005. Path enumeration by finding the constrained Kshortest paths. Transportation Research Part B 39, 545-563.

Varian, H.R., 1984. Microeconomic Analysis (Second Edition), W.W. Norton \& Company.

Watling, D., 2006. User equilibrium traffic network assignment with stochastic travel times and late arrival penalty. European Journal of Operational Research, 175, 1539-1556.

Wu, X., Nie, Y., 2011. Modeling heterogeneous risk-taking behaviour in route choice: A stochastic dominance approach. Transportation Research Part A, doi:10.1016/j.tra.2011.04.009.

Zhou, Z., Chen, A., 2008. Comparative analysis of three user equilibrium models under stochastic demand. Journal of Advanced Transportation, 42 (3), 239-263. 